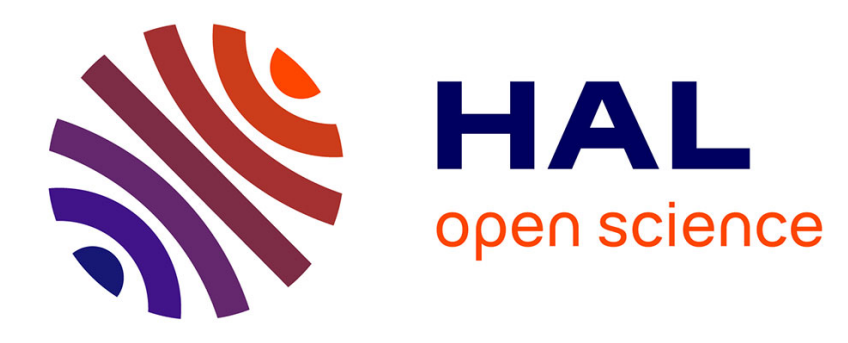

\title{
DU LANGUAGING AU SENS LINGUISTIQUE
}

Didier Bottineau

\section{To cite this version:}

Didier Bottineau. DU LANGUAGING AU SENS LINGUISTIQUE. Intellectica - La revue de l'Association pour la Recherche sur les sciences de la Cognition (ARCo), 2017, Langage et énaction: corporéité, environnements, expériences, apprentissages, 68, pp.19-67. hal-01818479

\section{HAL Id: hal-01818479 \\ https://hal.science/hal-01818479}

Submitted on 19 Jun 2018

HAL is a multi-disciplinary open access archive for the deposit and dissemination of scientific research documents, whether they are published or not. The documents may come from teaching and research institutions in France or abroad, or from public or private research centers.
L'archive ouverte pluridisciplinaire HAL, est destinée au dépôt et à la diffusion de documents scientifiques de niveau recherche, publiés ou non, émanant des établissements d'enseignement et de recherche français ou étrangers, des laboratoires publics ou privés. 


\title{
DU LANGUAGING AU SENS LINGUISTIQUE
}

\author{
Didier Bottineau \\ CNRS, FRE 2800 LDI (Lexiques, Dictionnaires, Informatique), Université Paris 13 \\ didier.bottineau@free.fr
}

\section{Résumé}

La cognition énactive rejette de la notion de représentation comme explication principielle et causatrice du fait cognitif. Ceci pose d'emblée un problème pour l'étude du langage humain, que l'on appréhende habituellement comme l'étude des rapports entre des formes symboliques, des pensées individuelles et un monde de perception locale et de connaissance générale. La présente étude explore ce que devient la notion de sens linguistique une fois repensée dans le paradigme de l'énaction et sans présupposer les notions de monde et de pensée comme faits matériels et spirituels autonomes démunis de genèse. Elle repart du languaging en tant qu'energeia et activité incarnée, interactive et située, et de sa contribution à la constitution de l'expérience de la vie ordinaire en tant que production de sens et de monde vécu, avec ou sans parole instantanée. Elle précise la différence entre le questionnement posé au langage par la linguistique, qui le constitue en systèmes et objets formels, et l'approche énactive, qui l'envisage comme processus incarné et situé. Elle propose que du fait de cet inscription, le sens linguistique doit être pensé comme un phénomène émergent et en voie de distinction, plus ou moins fondu dans la situation immédiate par son rôle pragmatique, et plus ou moins distingué en tant que création sémantique autonome ; et elle introduit une typologie des classes de sens linguistique selon leur degré d'inscription ou d'autonomie relatives et de regard réflexif porté par les sujets parlants. Elle précise comment certaines composantes linguistiques de la parole et du discours (syntaxe, prosodie, lexique, morphèmes grammaticaux), redéfinies dans leur dimension processuelle, participent à la constitution du sens, et esquisse des perspectives en typologie linguistique. La conclusion précise le sens du languaging en tant que vecteur de la co-évolution liant l'espèce à son environnement par son langage, ses langues et ses discours.

Mots-clés : languaging, embodiment, interaction, socialité, cognition, sens, sémantique lexicale et grammaticale, épistémique, pragmatique, lexique, morphologie, syntaxe.

\begin{abstract}
Enactive cognition rejects the notion of representation as an explanatory principle of cognitive activity. This immediately poses a problem for the study of human language, which is usually understood as the study of the relationships between symbolic forms, individual thought and a world of local perception and general knowledge. This study explores how the notion of linguistic meaning can be revised within the enactive paradigm and without presupposing the notions of world and thought as autonomous material and spiritual entities without a genesis of their own. It begins with languaging as energeia and embodied, interactive and situated activity, and details its contribution to the constitution of the experience of ordinary life as a production of meaning and empirical world, with or without the help of actual speech. It clarifies the difference between the analysis of language by linguistics, which views it as formal systems and objects, and the enactive approach, which considers it as an embodied and situated process. It proposes that because of this grounding, linguistic meaning must be viewed as an emerging and distinct phenomenon, which can be either merged with the immediate situation by its pragmatic role, or distinguished from it as an autonomous semantic creation; and it introduces a typology of classes of linguistic meaning according to their degree of relative inscription or autonomy and how speaking subjects reflect upon it. It specifies how certain linguistic components of speech and discourse (syntax, prosody, lexicon,
\end{abstract}


grammatical morphs), redefined as processes, participate in the constitution of meaning, and sketches out perspectives in linguistic typology. The conclusion elucidates the meaning of languaging as a vector of co-evolution linking the species to its environment through language, languages and discourse.

Keywords: languaging, embodiment, interaction, sociality, cognition, meaning, lexical and grammatical semantics, epistemics, pragmatics, lexicon, morphology, syntax.

\section{Introduction}

Le langage humain nous apparaît comme une machine à faire surgir du sens. Il semble intuitivement évident que les mots portent un sens, les phrases expriment une idée, les répliques d'un dialogue échangent des informations ou des instructions. Dans l'expérience de la vie ordinaire, la parole orale et le discours écrit sous toutes leurs formes se détachent de la situation ambiante et de l'action intégrante à la manière d'événements et objets saillants, attracteurs d'attention, vecteurs d'impressions et d'idées, de jugements et de projets, de questions et de consignes ; ils semblent concentrer dans l'interaction verbale et ses traces symboliques l'activité humaine de transmission intentionnelle du sens mental, la "pensée ", et reléguer à l'arrière-plan le monde ambiant en tant que substrat matériel objectif, intrinsèquement asémantique, candidat aux actes de représentation psychologique et offert à tous les actes imaginatifs. En particulier, la parole orale est par excellence l'activité incarnée qui ne laisse d'autre trace matérielle dans le monde que la manière dont elle a affecté intellectuellement et émotionnellement les consciences des participants à l'interaction verbale : elle ne vaut que par le «sens " qu'elle produit et semble accaparer le monde sémantique en l'expurgeant du monde ambiant, lui-même apparemment réduit à une masse physique, non porteuse d'esprit, vide de sens. De ce fait, il semble a priori légitime de s'intéresser au sens d'une réplique dans un dialogue ou d'une phrase dans un texte, de considérer la relation qui lie ce sens à la forme langagière qui en est responsable, de situer ce sens dans la dimension psychologique de " l'idée » ou de la "représentation », et de s'intéresser secondairement à la relation liant le domaine mental de l'idée au domaine matériel du monde physique. Telle est en tout cas l'illusion confortable et efficace entretenue par le discours convenu sur le langage ordinaire : nous vivons dans un monde matériel dont notre parole figure une idée qui le représente, le commente et le modifie au moyen de formes symboliques à l'agencement régulé ; le sens d'un mot, d'une expression ou d'un segment de discours est l'idée ou le fragment d'idée que ces segments encodent.

Derrière ces évidences, se cachent au moins deux problèmes. D'une part, le langage n'est pas que la production et l'interprétation de formes et traces évocatrices d'un monde idéel autonome, il est aussi activité incarnée et interactive située en pleine prise avec ce monde matériel dont on voudrait l'écarter à la manière d'un observateur ou témoin extérieur qui n'affecterait aucunement l'entité observée : la question du sens linguistique prend des contours radicalement différents selon que l'on s'intéresse aux traces formelles et règles d'agencement de l'activité langagière dans leur capacité à évoquer en tant que traces scrutées par un observateur, ou que l'on s'intéresse aux gestes de verbalisation réalisés in situ, in corpore et in actu par des agents parlants dont la parole concerte autant l'imagination d'un monde que l'action sur et dans le monde vécu. Le langage humain n'est pas qu'un jeu de formes encodant des idées, il est aussi une partie de gestes concertants dans une situation partagée dont on cherche à faire évoluer conjointement la compréhension et le contenu. On peut aborder le sens linguistique soit en observant des formes langagières dont on considère qu'elles ne figurent que des idées et reflètent le monde à la manière d'échos immatériels et inconsistants, soit en extrayant ces formes langagières des actes de parole consistants, incarnés, interactifs et situés, en prise directe avec ce "monde » matériel dont elles parlent, y compris les corps qui les réalisent et les esprits qui les suscitent et y réagissent. Le premier point de vue, de type spectatoriel, aboutit à l'idée d'un sens abstrait, idéel et disjoint du monde imaginé ; le second, d'orientation 
actorielle, tend à inclure la parole incarnée à la dynamique du monde matériel, et à remettre en cause l'idée d'un sens linguistique séparable, en contradiction flagrante avec les évidences mentionnées précédemment. D'où la première question que l'on adresse au sens linguistique : comment concilier l'idée d'un sens linguistique de type spectatoriel et immatériel, inconsistant, avec celle d'une activité langagière actorielle, consistante, inscrite dans le monde de matériel des objets et des corps qui la produisent et qu'elle perturbe ?

D'autre part, l'idée même qu'il y ait d'un côté un monde matériel et de l'autre sa représentation directe par la perception et retravaillée par le langage a été battue en brèche : avec ou sans langage, la réalité en tant que conscience d'un monde vécu résulte d'un processus continu de synthèse ; le monde apparent, où se distinguent le corps propre de chaque individu pour lui-même et l'espace ambiant en tant que théâtre d'interventions et d'interactions, est une figuration spécifique réalisée par la communauté de corps vivants d'une espèce donnée en un moment de son devenir évolutif. Cette figuration est pertinente matériellement et pragmatiquement parce que contrainte par les paramètres non visibles du monde physique en soi, mais son contenu est relatif au parcours d'autorégulation biologique qui caractérise la communauté de corps réalisant sa synthèse : elle constitue un Umwelt qui n'est pas une mimesis mais un modèle chargé de connaissance adapté à l'action de vie et de survie. Pour cette raison, le monde apparent, ce réel pris pour matériel et objectif, est lui-même sens, et se produit comme un fait cognitif ancré dans un processus biologique intangible, occulté par la discrétion de l'inscription corporelle des processus non langagiers, et masqué par l'évidence de l'inscription corporelle de l'activité langagière elle-même. Dans cette perspective, la synthèse du sens se distribue entre les processus intangibles de l'énaction générale et l'activité saillante de la parole immédiate réalisée dans le contexte de la première, laquelle conserve en mémoire le bénéfice des savoirs et savoir-faire enregistrés à l'occasion des épisodes d'interactions verbales antérieures. La question du sens linguistique va donc se décliner en deux versants : d'une part, la nature de la participation de l'activité langagière et de ses traces à la production d'un Umwelt humain, l'inscription de l'hominisation dans le languaging; et d'autre part, la capacité de l'activité langagière épisodique à générer un domaine de sens linguistique disjoint du monde matériel vécu, avec un effet de bicaméralisation de la conscience (dans un sens différent de celui de Jaynes 1976). Avant d'expliciter ce fonctionnement, rappelons en quoi « le réel est sens » en lui-même du point de vue de l'énaction.

Le paradigme de l'énaction définit la matière vivante comme de la matière physique animée par un processus dynamique dont la structure clôturée et ouverte sur son extériorité permet sa propre conservation. Cette conception fait écho au monisme matérialiste de Spinoza (1677/2005), en particulier la distinction natura naturans et natura naturata. Pour chaque espèce, ce processus d'auto-conservation évolutive est réalisée par des corps, échantillons de matière physique qui réalisent localement et momentanément les chaînes de processus opérationnels et causaux clôturés auxquels ils participent (Maturana et Varela 1980, 1987) : l'espèce, qui se manifeste comme une communauté d'êtres discrets et transitoires, se réalise par un processus continu d'entretien de ses unités actives et de génération d'unité ultérieures par inclusion de matière et consommation d'énergie; le corps réalise dans son enceinte la clôture opérationnelle qui s'ajoute aux lois du monde physique général et dont l'observation réflexive détermine la distinction d'une intériorité et d'une extériorité, constitutive de la possibilité d'interaction et de l'autonomie de l'agent vivant régulé par la dynamique de l'espèce qu'il réalise. Du fait de la clôture opérationnelle, les effets des interactions sont enregistrés en continu par la structure du corps vivant, dont la dynamique s'ajuste aux perturbations rencontrées, y compris celles qu'il a occasionnées par ses propres interventions : l'observation réflexive des enregistrements et leur comparaison aux interactions renouvelées motive des effets de connaissance mémorisée, d'apprentissage et d'anticipations constitutive de l'intentionnalité individuelle, et la coordination de cette dynamique entre les agents d'une espèce par la clôture opérationnelle qui les coordonne détermine une évolution conjointe de l'espèce, des perturbations qu'elle imprime dans son environnement, de l'environnement lui-même et de sa relecture en boucle par les agents de l'espèce, d'où la notion de co-évolution autopoïétique. L'autopoïèse des corps individuels au sein de chaque espèce, et de chaque espèce en tant que 
communauté de corps engagés dans un devenir d'apprentissage et d'auto-détermination, constitue les conditions d'auto-orientation intentionnelle à ces deux niveaux et implique que la vie est intrinsèquement un processus d'ordre cognitif (Stewart 1996, Bourgine et Stewart 2004). Chaque espèce réalisant un modèle évolutif de la distinction et du couplage intériorité / extériorité, l'observation réflexive de ce couplage par les vecteurs corporels de l'espèce constitue et énacte l'expérience d'un monde vécu en tant que relation entre le corps propre agentif autonome et l'environnement exploitable. De ce fait, les agents n'évoluent pas dans le monde en soi mais dans celui que leur clôture opérationnelle leur fait synthétiser et dans lequel ils opèrent la poursuite de leur survie en fonction d'une connaissance opératoire et pragmatique plutôt que d'une connaissance épistémique et réflexive telle qu'on la forge par le discours humain.

Dans ce cadre paradigmatique, l'approche biologique du langage humain va donc interroger la participation et l'originalité de l'activité langagière dans la cognition énactive à différentes échelles spatiales et temporelles, et à tous les niveaux d'inscription individuelle, interactionnelle et collective de ses réalisations. Cette approche concrétise le "potentiel épistémologique de l'energeia" aristotélicienne qui fonde la conception humboldtienne $(1836 / 1974)^{1}$ du langage humain comme activité constituante chez Coseriu (Bota 2012). Cette démarche permet de traiter à la fois la question de la plus-value énactive et de la discrétisation du sens linguistique dans l'expérience des interactions immédiates, et de mesurer son effet accélérateur sur la co-évolution du couple humanité / environnement. A cet effet, Maturana (1978) a forgé le concept de languaging, qui envisage comme activité éthologique d'auto-animation constituante l'ensemble des composantes du langage humain : le langage en tant que modalité de collaboration vocale hominisatrice caractérisante à l'échelle de l'espèce, la langue en tant que modèle de langage caractérisant le groupe humain, et la parole en tant que pratique d'une langue caractérisant les agents en interaction. Le languaging se définit comme matrice (Raimondi 2014ab) et opère par coordinations de coordinations récursives et observatrices de leur propre fonctionnement, qu'elles peuvent commenter et rectifier avec la progression historique des pratiques langagières; par son inscription dans les coordinations, le languaging prolonge l'interactionnisme social de Vygotski (1934/1997), Mead (1934/2006), Medvedev (1928/2008) et Volochinov (1929/2010) (cf. Bota 2012). Ceci implique que non seulement il n’y a pas de percevabilité du monde vécu qui ne soit profilé par le languaging en cours ou enregistré, mais également que le travail métadiscursif sur l'activité langagière permet à l'espèce hominisée de s'approprier le contrôle de la mutation continue de son propre domaine consensuel d'interactions: modifier intentionnellement et collectivement le modus operandi du languaging revient à reconfigurer le contenu des mondes vécus énactables par son intermédiaire, à faire évoluer notre idée du monde par notre parole, à en modifier la conscience et les projets, in fine à modifier le monde lui-même, toutes autres espèces comprises. Ce "monde» supposément objectif, que commente le discours humain, est lui-même un construit énacté, continuellement assisté par la matrice du languaging, y compris dans les moments vécus sans parole. Le «sens linguistique » en tant que domaine mental synthétisé par la parole et le «sens extralinguistique » de l'impression de réalité vécue à côté de l'acte de parole ou sans sa participation active s'interpénètrent mutuellement. La perception visuelle produit une visualisation, elle synthétise une fata morgana ${ }^{2}$ qui s'ignore (Frith 2007 ; la perçaction de Berthoz dans Berthoz et Andrieu 2011). Par la métaphore de la fata morgana, on insiste sur deux éléments : d'une part, la participation active, multimodale et non réflexive du corps dans la synthèse de l'impression de réalité (l'incarnation); d'autre part, la matérialité corollaire de l'impression résultante, qui s'ignore en tant que visualisation contruite. Du fait de l'ouverture de la clôture opérationnelle à l'entour, l'inclusion de signaux ambiants perturbe les organes sensoriels inscrits dans les motricités corporelles (Noë 2004, 2009 ; Gallagher 2005),

1 « en elle-même, la langue est non pas un ouvrage fait (Ergon), mais une activité en train de se faire (Energeia) » (Humboldt 1836/1974: 46).

$2 \quad$ Fata morgana : conjonction de mirages qui fait apparaître un élément de paysage matériel (île, falaise). De l'italien « Fée Morgane », à qui la tradition arthurienne celtique attribue le pouvoir de modeler des châteaux flottants sur les eaux en manipulant le vent. 
lesquelles sont distribuées et corrélatives (oculaire et visuo-faciale: Olivier 2011, phonatoire et visuo-faciale: Olivier et Bottineau 2015, auriculaire : Tomatis 1991; musculaire et neuronale : Berthoz 1997). Leur collaboration et son observation bouclée par le système cortical concourt à produire une scène de réalité où se distingue un corps vécu comme domaine privé, confiné et autonome, et un espace public, ouvert et indépendant, à 1 manière d'une scène offerte à l'intervention du corps privé : ce dernier n'est pas énacté que comme spectateur, mais comme acteur participant à la scène, laquelle n'est pas qu'un spectacle, mais aussi un atelier palpable (Merleau-Ponty 1945), un "opéracle » (Bottineau 2012b), un théâtre d'action muni de scenarios d'engagements possibles. La fata morgana présente est chargée de propositions d'actions futures parce que sa confection entrelace les enregistrements passés de l'historique continu de ses propres instants antérieurs. De ce fait, elle recueille la connaissance de routines communes et contraignantes, et se laisse profiler par les normes éthologiques et culturelles propre à l'espèce : la vision d'une « forêt » produite par le promeneur dépend de son vécu citadin ou arboricole au sein de sa communauté pratiquant le languaging comme matrice et champ de polarisation culturelle, y compris les usages du mot forêt dans l'univers culturel des discours communautaires où ce mot se trouve engagé dans l'expérience acquise du sujet. L'auteur de ces lignes est entraîné à perçacter la forêt et, indissociablement, énacter le mot forêt comme un espace de randonnée, alors que l'un de ses collègues Ivoiriens n'y voit qu'un espace infesté de prédateurs et auquel on ne se rend que pour y prélever une ressource indispensable (et ce collègue ne peut neutraliser ce ressenti dans une forêt française) : ces deux expériences divergentes, orientées par deux languagings au sein de deux mondes habités par deux communautés distinctes, témoignent de deux pratiques de la cognition énactive assistée par le languaging, et ne se rencontrent pas.

La parole instantanée ne peut donc pas être conçue que comme un formalisme qui encode des représentations du monde ou les idées que l'on s'en fait. Dans le vécu immédiat, elle est avant tout action et perturbation du "vent » qu'appréhende la fée Morgane du corps observatoire agissant pour constituer la fata morgana, et participe de l'auto-production du monde vécu. En tant qu'acte d'expression, la parole s'offre en spectacle, et se laisse distinguer en tant qu'activité productrice d'une fata morgana secondaire, réflexive, au sein de la première, non réflexive : le sens linguistique se vit aussi en tant qu'idée consciente d'elle-même au sein du monde en tant qu'idée qui s'ignore. Si la perçaction est un processus de figuration qui s'ignore, la parole se vit comme une activité de « conçaction » qui croit réfléchir et discuter le «monde réel » alors qu'elle transfigure le produit de la perçaction, quitte à en dissocier un objet attentionnel distinct. Enfin, la parole instantanée réplique ses propres épisodes antérieurs, les fait varier, les observe, les commente et les fait évoluer : elle participe au renouvellement continu de la matrice de coordinations de coordinations récursives qui réalise le languaging en tant que courant ou champ polarisé.

Cette conception où le fait spectatoriel émerge du fait actoriel implique le rejet des langues et formes de langues comme code (Stewart 2001, Kravchenko 2007, Linell 2013b), l'exploration du languaging comme pratique incarnée et distribuée (Erard 1998, Clark 1998, Kravchenko 2007, Cowley 2011ab, 2013, Thibault 2011, Bottineau 2011b, Linell 2013a, Raimondi 2014ab, Auchlin et al. 2004 pour la prosodie), l'explicitation de la socialité et de la pragmatique intégrée du sens linguistique (Rastier 1987, Itkonen 1997, Bottineau 2012c, Bondì 2015, 2016 et 2017), la co-construction du sens (Brassac et Stewart 1996), la co-constitution des sujets émergeant de l'interaction (participatory sense-making: De Jaegher et al. (2007), Cuffari et al. 2014, Froese 2016), l'interaction entre conditions de participation à une action et expression langagière (Barnabé 2017), et la nécessité d'une unification des approches sémantiques (Rastier 2006). Elle passe par le développement d'une bio-écologie énactive comme "fertilisation croisée de Maturana et Love " (Cowley, ce volume) concentrée sur la capture et l'orientation des engagements des agents par la dynamique de la matrice, avec notamment la distinction du languaging de premier et second ordre (Love 2004) - le second étant le creuset épiphylogénétique (Stiegler 1994) où s'imprime la dynamique matérialisée par le premier. L'activité participative du sujet parlant, est relativement hétéronome, ou autonome dans un système de contraintes flexibles ouvertes à la transgression créative à des échelles diverses (cf. Froese et al. 2011, Froese 2012). Le débat se concentre sur la 
pertinence de distinguer une ou deux fata morgana : un sens proprement linguistique, attaché aux formes symboliques distinguées du monde d'engagement comportemental, et le «sens » à la promotion duquel participe le languaging en tant qu'activité.

En grammaire et en linguistique des langues naturelles, les catégories descriptives et analytiques sont relativement peu contraintes par l'inscription bio-écologique de la matrice du languaging, ce qui complique l'introduction du paradigme au sein de la discipline. Si la parole est définie comme un geste minimal et confiné par le psychologue Pierre Janet, ses unités segmentales, les mots, ne sont pas définis par les linguistes comme gestes exécutables, mais comme signes percevables, avec le changement de posture phénoménologique que cela implique. Un modèle linguistique de premier ordre doit émerger de l'expérience des interactions (Kravchenko 2012), comme celui de Gardiner (1932), qui entreprend d'extraire une grammaire de la langue en modélisant les interactions verbales situées ; dans sa perspective multimodale et multicanal, un geste ou un clin d'œil peuvent produire un effet discursif ou y contribuer en association avec des manifestations verbales formalisées à proprement parler. Le paradigme énactif ne peut pas faire de la désignation et de la référence au monde extérieur au langage des primitives conceptuelles, mais doit les faire émerger de la distinction des fata morgana, avec comme extrêmes d'un côté le sense-making assisté par la parole, et de l'autre la parole en tant qu'activité de sense-making autonome, supplémentaire, en position d'observation, de commentaire et de création. Il doit concevoir le languaging comme un appareil éthologique d'auto-animation et de production de monde vécu dont le sens linguistique n'est qu'une composante émergente à relier à l'auto-production de l'individu sur la scène sociale, la gestion du groupe, l'inflexion du fait culturel, et le pilotage du languaging lui-même. La notion d'auto-animation concerne l'ensemble des catégories descriptives de la parole dans les langues : la morphosyntaxe et le lexique, les figements et la phraséologie, la prosodie et la gestualité. Elle consiste à revoir les formes-objets en schèmes-actes réalisés comme segments d'auto-animation, observés comme tels et comme traces symboliques, et vécus comme des évidences ergonomiques du fait de leur simplexité (Berthoz 2009, Bottineau et Berthoz 2015, Cowley 2016) ${ }^{3}$. Elle concerne également la production de modèles métalinguistiques de second ordre par des pratiques méta-discursives qui "décrivent », commentent et prescrivent des usages, et synthétisent des domaines de languaging secondaires qui conservent ou effacent plus ou moins les propriétés du premier ordre : l'auto-animation engagée, bio-écologiquement située. L'auto-animation de premier ordre vectorise la créativité (cf. Penelaud 2010 citant Bachelard 1948 ${ }^{4}$ ), y compris sa propre auto-créativité, avec les conséquences que cela suppose pour l'auto-orientation de la co-évolution espèce / environnement, son inintelligibilité et/ou sa manipulabilité du point de vue des agents ${ }^{5}$; en second ordre, elle sous-tend l'auto-organisation des langues par la sélection darwinienne des pratiques (Oudeyer 2006) et, in fine, la générativité des modèles formels et calculatoires. Pour explorer sa participation au sense-making, on repart donc de la manière dont une théorie linguistique produit son questionnement sur le sens (section 1), puis on explore le processus de distinction du sens linguistique à l'échelle de la parole (section 2) avant de réengager la même démarche dans la distinction de certaines de ses composantes que sont la syntaxe (section 3) et les marqueurs lexicaux et grammaticaux (section 4).

\section{Comment une théorie linguistique produit son questionnement sur le sens}

3 Un observateur de second ordre qualifie de simplexe une relation agent / environnement vécue comme ergonomique du fait de l'adéquation de la complexité incarnée à la complexité du monde produit qu'il s'agit d'explorer et de manipuler. Parler est vécu comme non problématique en dépit de la complexité des interactions verbales et des participations modélisées en second ordre. 4 "Le langage est aux postes de commande de l’imagination. » (Bachelard, 1948, p. 8) 5 Exemple de slogan publicitaire qui intervient directement sur la planification du reprofilage du domaine consensuel d'interactions : Demain, chaîne alimentaire et chaîne d'approvisionnement ne feront qu'un (HSBC). 
Le langage humain est traditionnellement pensé comme une faculté d'exprimer des "pensées » et de les communiquer. La question de la relation entre "langage » et "pensée " a été discutée en philosophie mais peu explorée en linguistique compte tenu du cloisonnement disciplinaire initié par Saussure ${ }^{6}$ : la langue est pensée comme structure permettant de décomposer la pensée prélinguistique confuse en éléments discrets et distincts (Saussure et Guillaume), et comme machine à formaliser la synthèse « d'actes de représentation » (Guillaume 1964, 2003, 2004, 2007). Ces actes ne sont pas la représentation diplomatique d'un monde dit "extralinguistique », mais la représentation psychologique d'un sens vécu comme transfiguration stabilisée d'une intuition confuse et fugace. L'opérativité de la production de discours contribue à la présentation consciente d'un effet de sens qui ne correspond pas à une représentation préalable ; mais les formes langagières sont encore pensées comme symboles abstraits plutôt que schèmes d'action incarnée, la réplication reste ignorée, et le fait social demeure relativement marginal, bien qu'existant ${ }^{7}$, tout comme en linguistique cognitive (Croft 2009 ; pour les grammaires de construction, Desagulier 2011).

Pour cette raison, le sens linguistique est en partie pensé hors de l'inscription qui lie les actes de parole ou d'écriture à une situation écologique (le panonceau instructionnel à la piscine; le texte imprimé dans le livre en tant qu'institution normée selon le type de livre). Sur cette base, la sémantique part de l'étude des correspondances entre les énoncés transcrits et leur interprétation à la lecture, et s'intéresse à la décomposition des textes en signes qui participent aux distinctions. Cette démarche sépare la sémantique, l'étude du sens produit par les formes langagières, de la pragmatique, le calcul des intentions, présupposés, conséquences et implicatures conversationnelles du sens linguistique littéral une fois qu'on le ré-inscrit dans un contexte bio-écologique constaté (conversation orale) ou simulé (textes écrits). La linguistique s'est donné pour objet empirique les formes symboliques d'une éthologie propre à l'espèce humaine plutôt que l'activité elle-même, marginalisant l'oralité et la pragmatique comme des périphériques : elle est affectée par un written bias décontextualisant (Linell 2005, 2012), même quand un contexte imaginaire est invoqué. Cette tendance est modérée par la pragmatique, qui se concentre sur certaines facettes de l'inscription, et certaines théories, qui incorporent à leurs modèles abstraits de deuxième ordre des paramètres de premier ordre : la corporalité multimodale et les schèmes-images gestaltiques de la linguistique cognitive ; et dans certaines théories de l'énonciation le dialogisme, la polyphonie, (Bakhtine 1970, 1978 ; Linell 2009 ; Bres et al. 2005, Vion 2014), la médiativité (Guentcheva 1994), la polylectalité (Berrendonner et al. 1983), ou la sémantique interprétative de Rastier. Une communauté scientifique particulière produit des savoirs et objets de connaissance en interagissant et constituant son propre domaine consensuel d'interactions (Mondada 2005), et la linguistique n'échappe pas à la règle (Culioli 1982, Valette 2006). Cette dynamique d'auto-exclusion peut poser problème dès lors que les construits terminologiques et conceptuels, phraséologiques et idéologiques, doivent être diffusés à l'extérieur de la communauté de languaging qui les a produits : le grand public est censé percevoir, mais pas «comprendre", que la chicane, le ralentisseur, le plateau et le dispositif prudentiel du discours technocratique des panneaux routiers désignent en fait des obstacles intentionnellement accidentogènes pour l'automobiliste qui n'a pas ralenti à la vitesse prescrite (avec les conséquences juridiques que susciterait cette analyse si elle devait être retenue par un automobiliste effectivement victime d'un accident) ; la voie de stockage est censée désigner ce que les moniteurs de conduite appellent communément un tourne-à-gauche; dans l'enseignement de la grammaire, l'opérateur a désigné ce que l'instituteur nomme traditionnellement l'auxiliaire, sans que la compréhension du terme par les élèves ou par l'enseignant lui-même ne fasse réellement débat.

\footnotetext{
$6 \quad$ Sur les hésitations de Saussure et Benveniste dans le traitement de la question langage / pensée, cf. Bergounioux (1995).

Tollis (2008, 2017) interroge toutefois l'émergence chez Guillaume d'une « socio-opérativité ».
} 
Comme toute discipline scientifique, la linguistique est une pratique communautaire visant à construire collectivement des objets de connaissance novateurs en recourant à un protocole technique régi par un discours, une phraséologie et une terminologie négociés et convenus. Mais dans ce cas particulier, les objets produits par le discours sont censés modéliser les processus mis en œuvre par l'activité de discours elle-même, dans le cadre d'une démarche réflexive traditionnellement qualifiée de métalinguistique : la pratique de la linguistique est un languaging de second ordre, restreint et contraint par ses propres règles de choix thématiques et procédures discursives, et il est relatif au languaging de premier ordre, celui que pratiquent, comme tout le monde, les linguistes quand ils parlent d'autre chose que du langage. Par définition, ce languaging de second ordre, spécialisé dans l'observation du premier, s'en distingue et se munit d'une régulation autopoïétique qui implique une dérive inévitable par rapport à celle qui prévaut au sein du premier ordre ; et la question est de savoir si le second se dote de règles et contraintes internes explicites de nature à limiter ou compenser cet écart : le métadiscours (discours spécialisé relatif au discours général) doit s'autoréguler par un patadiscours (méta-métadiscours, ou métadiscours secondaire et réflexif, relatif à lui-même en tant que métadiscours) ${ }^{8}$. De fait, le méta-discours linguistique tend à analyser les processus langagiers de premier ordre (la parole incarnée et située, l'intonation et la gestualité interactives) en objets analytiques de second ordre (classes de morphèmes, mots et syntagmes ; fonctions syntaxiques ; catégories sémantiques : temps, aspect, modalité, définitude, évidentialité...) sans expliciter la manière dont on transite de la perspective actorielle non réflexive du premier ordre à la perspective spectatorielle réflexive du second en tentant tout de même d'y préserver, modéliser et analyser les paramètres constitutifs du premier, à savoir l'incarnation et l'interactivité - ce qui ne peut être réalisé que par une démarche patadiscursive. Le métadiscours linguistique engendre également son questionnement : compte tenu du processus historique de segmentation de l'écrit, de l'évidence apparente de la relation mot / chose, de l'idée qu'une phrase se génère ou se produit comme on façonne un objet à partir d'éléments, la question du mot, rendue visible et cruciale par l'acte d'observation même, a largement servi de point de départ à la problématique langagière (Quine, Peirce; Saussure, Guillaume). L'observation du mot-objet isolé induit la distinction du signifiant phonique et du signifié conceptuel ; l'adoption d'une posture spectatorielle (cf. "image » acoustique) relègue la motricité du mot-acte à l'arrière-plan constituant le signifiant sans entrer dans sa définition (Piotrowski 2012) ; l'invention du signifié comme concept général et abstrait entraîne la nécessité de sa recontextualisation par une invention supplémentaire, l'idée d'un référent corrélable à une chose du monde et à une chaîne de discours. Comme on le constate, la posture spectatorielle en languaging de second ordre expurge le mot-objet des paramètres "incarnation » et "interactivité » propres au mot-acte vécu en languaging de premier ordre, et rend nécessaire leur réintroduction ultérieure, quitte à faire proliférer des distinctions nécessaires pour le linguiste mais peut-être non pertinentes pour les acteurs du languaging initial. La théorie du mot-objet ignore ou marginalise la réalisation du mot-acte comme schème phonatoire perturbateur de l'interaction, promoteur d'auto-animation distribuée aux interlocuteurs; elle occulte le jeu des intégrations motrices et sensorielles multimodales réciproques (motricité de la perception auditive langagière : Liberman et Mattingly 1985 ; Bottineau 2017). Toutefois, il faut reconnaître que cette démarche s'imposait

8

Le préfixe pata- (de pataphysique, Jarry 1897-1898), au signifiant ludique empreint d’auto-dérision, mais au signifié des plus sérieux, contracte la construction néologique

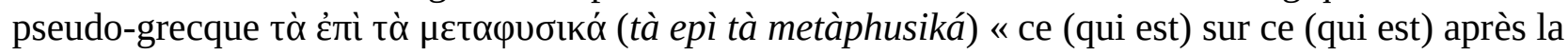
physique » : il exprime le redoublement de la prise de recul réflexif de type meta- et opère littéralement l'adjonction d'un commentaire de second niveau appliqué à un commentaire de premier niveau lui-même appliqué à l’observé, la « nature ». Malgré son allure peu conventionnelle, ce préfixe condense l'idée d'une réflexivité récursive dont les boucles réitérées constituent les conditions d'observabilité et contrôlabilité de sa propre dynamique et de ses effets, et désigne adéquatement l'exigence épistémologique requise pour produire un méta-languaging qui ne diverge pas de son propre propos. 
historiquement pour le mot objet pour diverses raisons : (i) la démarche scientifique consiste à commencer par distinguer et classer des parties et unités au sein d'ensembles et de " touts »; (ii) le mot-chose préexiste comme observable du fait de l'écriture, acte et symbole métadiscursif historique et civilisationnel avec sa dimension métalinguistique et transfiguratrice relativement à l'oral (la raison graphique de Goody 1980); (iii) les théories lexicalistes s'appuient sur le mot comme unité de travail pour les locuteurs, considérant qu'on ne mémorise pas les énoncés, mais leurs constituants et les règles d'assemblage (structures syntaxiques, constructions, exemplaires des modèles analogiques de Lavie (2003) et Itkonen (2005), ou modèles de valence lexicale, statique avec Tesnière (1959), dynamique avec la théorie de l'incidence de Guillaume 1964). Cependant, toutes ces approches ont un caractère autopoïétique, déterminé de l'intérieur par leurs règles et techniques de production d'observables de second ordre, et elles ne semblent pas s'être munies d'un processus d'auto-contrôle "patadiscursif » qui les prémunisse contre les risques d'une inflation conceptuelle déterminée par la neutralisation des paramètres fondateurs de l'incarnation et de l'interactivité. Chaque théorie résulte d'une dynamique qui fédère une communauté par un domaine consensuel d'interactions spécialisées qui délimite et légitime un terrain d'action et de production intellectuelle concertée où s'opèrent des distinctions spécifiques. Reposons donc la question du sens linguistique et de sa distinctivité en réintégrant les principes qui président à son émergence, à savoir : la matrice du languaging comme composante semi-distincte de la clôture opérationnelle ; la participation des agents par orchestration d'actes auto-animateurs ; l'émergence de distinctions morphosémantiques de niveaux de résolution et contenus divers selon l'attention portée sur les engagements, leurs produits et leurs effets aux différents niveaux de participation dans l'espace, le temps, les réseaux d'agentivité.

\section{Distinguer la sémantique langagière en tant que monde vécu en voie de séparation}

On a rappelé que l'expérience de la réalité immédiate relève de la production d'une fata morgana polarisée par le languaging occulte : le réel, ou la "réalisation » au sens de l'anglais realization "prise de conscience ", est un processus non réflexif (aveugle à lui-même) de synthèse d'une figuration perçactée en fonction de routines procédurales contraintes, normées et commentées par la matrice langagière. Parler à un moment donné, c'est renouveler par l'action phonatoire contrôlable et observable les actes d'auto-animation constitutifs de la matrice qui oriente la perçaction. De ce fait, l'effet produit par l'acte de verbalisation peut s'inscrire entre deux extrêmes : soit la prise de parole n'est pas discernée comme événement autonome, et elle se contente de participer à la modification de la fata morgana ambiante par ses effets pratiques et sans dissocier de sens spécifiquement linguistique ; c'est le cas de l'interjection oups, qui participe de la co-construction et co-gestion d'une interaction matérielle en cours sans éveiller en elle-même une représentation sémantique isolable. Soit l'attention se concentre sur la spécificité des effets de l'auto-motivation langagière : "Tiens, il pleut », déclaration qui dégage en elle-même une idée distinctive, et relègue en arrière-plan la fata morgana ambiante, le "monde », le "contexte », "les choses », "le référent », tout en mettant en exergue une fata morgana supplémentaire, saillante et distincte, le "sens linguistique » avec ses effets scéniques. Dans ce cas, le monde idéel exprimé par le "contenu » la parole est éprouvé comme distinct du monde matériel qui contextualise l'acte de parole, avec des effets momentanés de "bi-fenêtrage » ou compartimentage attentionnel et des relations de type désignation, dénotation, connotation, référence, indexicalité, évidentialité, mondes possibles. Ce continuum entre indistinction et distinction du sens linguistique situe l'effet pragmatique au premier plan et l'effet épistémique au second: l'interaction verbale fait prioritairement sens par la manière dont elle fait progresser la co-énaction de la situation en cours, et fait secondairement sens en elle-même si l'acte de parole focalise les attentions sur la mise en scène qu'elle vectorise en la séparant de la situation en cours, par prise de recul observatrice et réflexive ; la sémantique couvre l'ensemble du spectre de la parole opérative à la parole spéculative. Des degrés de cette distinction émergente dépend la transition d'un couplage d'interlocuteurs en 
mode actoriel à un couplage d'interlocuteurs en mode plus spectatoriel (toujours sur fond actoriel) et d'une parole opératoire à une parole observatoire, ou dont l'opérativité se boucle sur elle-même et produit un monde vécu domanial qui peut être ressenti comme détaché de l'inscription. Il en résulte une Gestalt où le sens linguistique se manifeste comme une saillance émergeant d'un fond non verbal. La question est de savoir jusqu'où il faut aller dans cet acte de distinction de sémantiques de premier ordre et de second ordre, l'une du point de vue de l'agent de parole, l'autre de celui de l'observateur méta-discursif qui modélise le premier. A la suite de Kravchenko (2011), on revoit donc la distinction sémantique / pragmatique dans un cadre orientationnel. En sémantiques de premier et second ordres, trois cas se présentent :

(i) en premier ordre, le sens pratique : l'agent parlant ne distingue pas l'effet de sens propre à la formulation verbale de la progression de la collaboration située ; en second ordre, le métadiscours reconstruit un sens pragmatique, pensé comme distincts d'un sens épistémique construit comme objet de connaissance. Exemple : dire «oups » et coordonner l'interaction entre agents autour de l'événement imprévu.

(ii) en premier ordre, la distinction des effets de sens scénique et pratique : l'agent parlant scinde plus ou moins l'interprétation entre un effet de sens où s'imagine une scène langagière référençable ou imaginable, et une relation d'inscription à monde vécu qui contextualise la parole (impulsion d'action, implications, pertinence) Exemple : dire " les bus sont toujours en retard, pas vrai ? » en entreprenant un acte de séduction à un arrêt d'autocar. En second ordre, ce cas est le siège de la distinction sémantique / pragmatique, mais il vaudrait mieux parler de sémantique épistémique (la scène comme objet de connaissance avec effet de transmission d'information pour l'observateur) et de sémantique pragmatique (l'objet de connaissance comme question et stimulus d'une réponse inférentielle).

(iii) en premier ordre, le sens onirique : l'agent parlant produit un effet de scène imaginaire non plausible, non figurale, incohérent ou non reliable au monde vécu comme non langagier : Colorless green ideas sleep furiously. En second ordre, l'effet de sens est reconstruit comme poétique par l'analyste. Pour leur part, les linguistes fabriquent également des énoncés à sens théorique, dont l'effet scénique se voudrait non situé en premier ordre : Le chat mange la souris. Tout lecteur conçoit la scène théorique figurée par cette déclaration simulée et convenue même si personne ne dit ni n'écrit jamais une phrase pareille en ces termes (en dehors d'un cours de grammaire sur la voix active), et surtout pas dans une situation ou un chat dévorerait effectivement une souris. Ces effets de sens onirique et théorique sont vécus en premier ordre comme dégagés de la situation parce qu'ils défocalisent l'attention de leur participativité à la fata morgana ambiante, mais en second ordre on leur reconstruit bien une portée pratique, comme : tester l'autonomie de la syntaxe générative, promouvoir une cause théorique, faire vivre une expérience esthétique au sein d'un groupe dédié, etc.

\section{Le sens pratique}

En épisode épidiscursif de languaging de premier ordre, une verbalisation produit un effet de sens pratique lorsqu'elle accompagne et coordonne les actions des collaborateurs impliqués. Il s'agit principalement des interjections, difficiles à classer en second ordre (Barberis 1992, Rosier 1995, Buridant 2006), dépourvues de mots lexicaux catégorisés (noms, verbes), pour lesquelles ont ne peut pas isoler d'effet de sens scénique spécifique autre que le modèle paradigmatique de situation potentiellement concerné. Chaque interjection peut être glosée par le modèle de collaboration qu'elle convoque: Oups! survient comme par réflexe conditionné lorsqu'un agent commet un agissement jugé en infraction par rapport à une attente commune (renverser du café à côté de la tasse), le jugement étant convenu et attribué indistinctement à l'ensemble des participants sans différenciation de points de vue subjectifs (tout comme les enfants observés par Vygotski produisent de la parole efficace qui ne suppose pas la distinction des agents du groupe); mais l'appropriation réflexive de l'interjection en fait un outil de mise en scène de soi dans les rapports intersubjectifs (Barberis 1995, Swiatkowska 2000). Quand oups survient dans son usage behaviorien, le locuteur peut tenter de bloquer ou retarder le réflexe, comme le candidat malheureux aux primaires 
républicaines de la présidentielle américaine de 2012, Rick Perry, lors d'un débat où il ne retrouvait plus quelle agence gouvernementale il programmait de supprimer ; on peut tenter de neutraliser son effet collaboratif, comme dans le film Independence Day, lorsque le pilote terrien de l'OVNI alien part en marche arrière ("oops ») et que son copilote lui fait dédire le oops de manière à invalider la reconnaissance de l'erreur et la faillibilité de l'agent qu'elle implique (pour une analyse détaillée, cf. Bottineau 2013d). On peut même se mettre en scène comme juge et rendre un verdict sur l'action d'autrui en "oupsant » à par procuration (exemple d'une collègue voyant arriver un collègue portant une cravate au goût discutable : «oups !"); cette pratique s'est popularisée au point que l'interjection a été prise pour titre d'un magazine "pipole » international publié dans diverses langues (Oops !, Oups!), spécialisé dans le voyeurisme et la stigmatisation des agissements des célébrités - et en retour, l'apparition de ce titre a dû contribuer à en renforcer l'usage. Ces emplois attestent de la diversité des points de vue à partir desquels l'infraction est observable, ce qui fait du marqueur un opérateur de participatory sense-making (De Jaegher et al. 2007) en réseau de participations hétérogènes et caractérisé par la non-localisabilité (Cowley 2007a, 2009). Le verbe néologique oupser, fréquent sur les réseaux sociaux, atteste de la mise en observation réflexive de la pratique dans le cadre de la récursivité du languaging, ce qui constitue l'acte en élément scénique et convertit l'interjection oups à effet de sens pratique en lexème oups- candidat à la distinction des effets de sens scénique et pratique, reconstruits comme épistémique et pragmatique en second ordre. L'observation réflexive des interjections et pragmatèmes (cf. infra) se manifeste par des répétitions hiérarchisées et catégorisées (oups de chez oups), des adverbes de commentaire (carrément oups), des segments de discours métalinguistique intégrés dans l'épilinguistique (Tiens, j'ai dis tiens ! dans le disque Jacques Crabouif Higelin, 1971) ${ }^{9}$.

L'emploi de chaque interjection active un modèle de coordination intersubjective et distribue des rôles différenciés selon la nature de la participation de chacun dans l'action engagée : bigre pour la détection d'une situation déconcertante pour le sujet comme pour le groupe, qui paralyse la progression de l'action individuelle et collaborative; zut pour le dépit et l'invective qu'il peut entraîner (selon inscription écologique avec la gestualité, le regard, l'intonation); bof pour l'indifférence blasée, etc. Il est très important de souligner que si ces interjections semblent exprimer des réponses émotionnelles subjectives à des stimuli matériels objectifs, elles s'emploient surtout en situation d'interaction et, mêmes employées par un locuteur isolé, elles lui font exécuter un modèle de performance émotionnelle conforme à une norme collective, ancrée dans et distribuée par les interactions verbales du languaging fantôme enregistré : elles n'ont rien d'idiosyncratique. Cosnier (1986, 1987), Auchlin (1995) et Plantin et al. (2000) insistent sur la socialité des émotions, à la fois par leur conformité à des normes et par l'inscription interactive de leurs opérateurs langagiers. Ces marqueurs, que l'on formalise comme linguistiques par réduction au mot-objet écrit, opèrent en premier ordre comme mots-actes bio-écologiquement situés : leur exécution requiert les paramètres intonatifs, prosodiques, accentuels, rythmiques, gestuels habituellement pratiqués, qui permettent de faire varier les réseaux participatifs, comme zut de dépit réflexif ou d'invective adressée ; il s'ensuit des effets de polysémie apparente pour le linguiste qui traduit le mot-objet de second ordre en en extirpant les paramètres incarnés et relationnels de la réalisation du mot-acte. Certains mots lexicaux, normalement désignatifs et évocateurs d'une image scénique séparable de la situation de parole, sont dits pragmaticalisés dans certains de leurs emplois du fait de leur participation à la gestion de la situation en cours et de l'impossibilité de leur attribuer un référent distinct, comme bon, tiens, tenez, va, allez, quoi (nordisme), mais final (normandisme Chuis fatigué, mais $!^{10}$ ) : contraints par une inscription interactive munie de paramètres bio-écologiques, ils produisent une impulsion collaborative sans éveiller d'effet scénique correspondant à celui qu'éveillerait le même mot-objet exécuté comme autre mot-acte dans d'autres engagements - cf. un bon vin vs allons bon!; Va te coucher vs Pauv'type, va! Quoi de neuf vs Rien de neuf, quoi!

\footnotetext{
9 https://www.youtube.com/watch?v=dRjozdfB2xE

10 Source : Daniel Roulland, usager coutumier et commentateur de cette construction dans nos conversations personnelles.
} 
Tenez! (prenez ceci) vs Tenez, pas plus tard qu'hier, (...), la théorie des genres vs ouais ! Genre!. Certains de ces mots-objets, comme tiens, présentent un paradigme très ouvert d'inscriptions et de réalisations divergentes du mot-acte, au point de sembler de former un réseau de sens polysémique à la limite de l'incohérence (du point de vue adopté en second ordre).

Le sens pratique est également particulièrement représenté dans la sémantique des formes grammaticales. Considérons deux cas très différents, la morphosyntaxe de l'interrogation et l'emploi des temps verbaux dans diverses langues.

En japonais, la particule finale $k a$ «marque l'interrogation » : elle transmet le relais énonciatif à l'allocutaire et gère la participation des interlocuteurs à la promotion conjointe de la production d'un monde vécu ensemble. En français, trois structures interrogatives gèrent trois modes de coordination : est-ce que pour la transmission informelle (Est-ce que tu en veux?), l'inversion du verbe et du sujet pour la transmission formalisée (En veux-tu?), et la montée intonative pour une transmission inopinée et motivée par une impulsion émotionnelle (surprise, intérêt, exaspération selon les autres modalités d'inscription : Tu en veux ? cf. le sketch T'en veux ? de Smaïn fumant et offrant un joint ${ }^{11}$, avec montée progressive, articulation confuse et regard égaré). L'effet de sens pratique est lié à la forme même des structures : la fluctuation intonative est orientée par l'impulsion émotionnelle; l'inversion verbe-sujet est une démarche sophistiquée qui demande une attention particulière à l'accord verbe-sujet et souligne l'effort relationnel consenti envers l'autre par l'effort de structuration ; le pragmatème est-ce que, souvent figé sous la forme eske dans la transcription de dialogues informels, amorce la question de manière anticipée et facilite la co-gestion de la transmission du relais dans l'interlocution; cet ajout de complexité apparente opère en fait un détour simplexe en introduisant un opérateur de facilitation et de convivialité dialogale.

Dans le domaine de l'expression de la temporalité, les théories de l'énonciation et de l'interlocution ont montré comment les temps verbaux ne figurent pas le temps en tant que représentation mentale topologique, mais mettent en scène des configurations dialogiques et dialogales où se construit le rôle du locuteur dans sa dimension modale (l'autorité du locuteur face au groupe en parlant au futur et au passé simples) et énonciative (le locuteur comme médiateur d'une parole réitérée et évolutive dans la médiativité de Guentcheva 1994), et où se différencient les points de vue interlocutifs par un jeu de configurations (Douay et roulland 2014). Le temps chronologique lui-même, de type schématique, est explicité lexicalement par des expressions ad hoc (hier, il y a un mois...) qui donnent accès à des localisations et segments de durée diversement positionnés sur l'idée normative d'un temps linéaire irréversible fragmenté en époques: cette phraséologie pratiquée dans les interaction verbales réitère un modèle commun d'énaction du temps qui permet de coordonner les actions relativement à un système de repérage partagé ; certaines langues pratiquent des modèles de coordination temporelle très différents (circulaires, non linéaires). Ces formes n'encodent pas des représentations mentales intériorisées par les sujets ; au contraire, leur pratique dans l'interaction située coordonne les agents sur la participation conjointe à des mises en scènes répliquables, dont l'enregistrement et la récurrence créent des effets de représentation distribuées dans l'interaction, non localisables. Núñez et Sweetser 2006 ont montré dans une étude de terrain comment en Aymara (altiplano péru-bolivien) la représentation du futur comme espace localisé à l'arrière du corps parlant et non à l'avant (hors du champ visuel, domaine de la connaissance) se construit de manière distribuée et émergente par une coalition de formes verbales et de gestes ancrés dans l'interaction verbale.

Les langues se différencient par des systèmes grammaticaux morphosyntaxiques dédiés à des mises en scènes coordinatives divergentes : le breton et l'inuktitut disposent de modes verbaux permettant au locuteur de s'afficher comme parlant au nom de la communauté et de légitimer sa propre position en adossant son propos à un modèle coutumier ( $\mathrm{Pa}$ vez klañv, ez a d'ar medisin " quand on est malade, on va chez le médecin ", où vez est au mode dit fréquentatif et présente le locuteur comme le répétiteur d'une vérité convenue, produisant un effet comparable à celui de l'emploi général de on français); l'imparfait des langues romanes permet au locuteur médiatif de se 
présenter comme participant à un continuum narratif dialogique (Le 26 avril 1986 explosait le réacteur numéro 4 de la centrale nucléaire de Tchernobyl, stéréotype de l'introduction journalistique); les langues bantoues différencient des temps du passé et du futur pour des événement vécus individuellement ou correspondant à la temporalité collective de la communauté ; le basque présente une conjugaison allocutive tutoyée qui réserve expressément la prise de parole à un destinataire ciblé et affiché comme tel devant témoins (ederra da "c'est vrai », ederra duk / dun «c'est vrai » adressé à un homme / une femme); la conjugaison des verbes tagalog (Philippines) et breton focalisent l'attention sur l'un des arguments du verbe ou de la phrase ; les langues romanes et l'anglais présentent diverses formes progressives $($ be + -ing, être en train de, estar/stare + gérondif) qui permettent de coordonner des configurations de points de vue relativement à la scène considérée dans ses dimensions pratique et scénique (Bottineau à paraître); les langues romanes coordonnent les attentions relativement aux notions lexicales par le système de l'article, ce que ne font pas le russe et le japonais. Ces "grammaires» de second ordre sont symptomatiques de pratiques de coordination de premier ordre tellement prégnantes qu'elles se réalisent par des opérateurs stylisés omniprésents à tous les instants de l'énonciation, et qu'il s'agit de s'approprier par participation pour l'apprentissage de langues secondes. Par exemple, une grammaire du second ordre dit du passé simple français qu'il représente le temps d'événement de manière perfective, en visualisant l'intégralité de son déroulement du début à la fin, ce qui lui permet d'enchaîner des narrations. La chose est vraie du point de vue spectatoriel du lecteur qui analyse la forme sans se jouer la mise en scène déterminée par son emploi, mais passe à côté de l'essentiel : en français, le passé simple opère un acte de langage présent par lequel le locuteur investit l'autorité de l'expert autoritaire capable de décréter ce que le passé est pour la communauté, à l'exclusion de toute autre version émanant d'un locuteur rival possible ; pour l'employer, il faut donc être historien (inventeur du passé officiel) ou écrivain (inventeur de passé fictionnel), et la réussite de l'acte de langage au passé simple (la felicitousness austinienne) dépend de la satisfaction de conditions culturelles et sociales, exactement comme pour la promesse, l'acte de baptême ou la cérémonie du mariage. Ce cas illustre en quoi une grammaire énactive de premier ordre reformule l'effet du temps verbal en tant que mise en scène d'un acte social introduit par un locuteur et participant humain au sein d'un groupe engagé dans l'action concertée et laisse émerger un effet de représentation énacté à partir de cette mise en scène, sans présupposer de représentation prédonnée à encoder. Toute la grammaire de second ordre de la description des langues a vocation à évoluer vers une grammaire du premier ordre qui rende compte des opérateurs grammaticaux en termes d'actes coordinatifs et énactifs : l'emploi de tout marqueur grammatical engage un groupe d'acteurs dans un jeu de rôles dont émerge un effet scénique. Une grammaire abstraite de second ordre, qui étudie les formes hors des interactions verbales situées, mais auto-contrainte par l'interactivité des paramètres du premier ordre va modéliser des faits de langue comme les temps verbaux ou les déterminants en termes de modèles d'interprétation dramatique (Bottineau 2014b), à la manière du texte théâtral qui préfigure la performance (Ubersfeld 1977). On peut alors différencier l'apprentissage de la langue première, avec des locuteurs enfantins qui s'engagent dans une participation directe au jeu d'acteurs vectorisé par les formes sans passer par leur introduction théorique dans le cadre d'un discours grammatical, et l'apprentissage d'une langue étrangère ou seconde, qui passe souvent par la présentation théorique de l'effet de ces formes, formulé en termes instructionnels (à l'instar de la direction préparatoire du jeu théâtral à partir du texte écrit) compte tenu de la démarche planificatrice que cela suppose. Par exemple, l'article défini peut être présenté comme un opérateur avertissant l'auditeur qu'il dispose d'un enregistrement accessible du référent correspondant au nom introduit par l'article (passe-moi le sel) alors que l'article indéfini produit l'avertissement contraire (il y a un problème) : la question abstraite de la définitude émerge de la différenciation concrète des points de vue en interaction relativement à un objet autour duquel se négocie la conjonction des attentions engagées dans l'interaction verbale. 
L'expérience ordinaire des énoncés en premier ordre d'expression du languaging tend à faire discerner un sens scénique d'un sens pratique : J'ai paumé les clés du camion ${ }^{12}$. Ce couplage de premier ordre varie en fonction de ce que peuvent construire les participants à l'acte de parole selon leurs points de vue : les agents imaginaires censés connaître le personnage joué par Coluche dans son contexte (le camion, René, Zézette, l'armoire) matérialisent une scène précise avec des effets pratiques déterminés ; les spectateurs du Schmilblick qui voient l'acteur Coluche faire irruption dans le spectacle du jeu en jouant ce personnage qui détourne la diffusion à l'antenne dans ses propres intérêts construisent un couplage totalement différent du fait d'une inscription plus stratifiée. Ces deux couplages ne coïncident pas avec la distinction des effets épistémique et pragmatique en second ordre que l'on peut discerner à partir de la version écrite décontextualisée j'ai paumé les clés $d u$ camion, qui évoque un modèle stylisé de situation scénique associé à un modèle stylisé d'effets pratiques. L'écrit ne donne à voir que la forme graphique des mots-objets et ignore la réalisation des mots-actes dans leur inscription bio-écologique : à partir de ce qu'il donne à voir, il permet la construction directe d'un modèle paradigmatique des effets scéniques possibles, ce qui donne l'effet de sens épistémique comme objet de connaissance construit et clôturé. Compte tenu de ce que l'écrit ne donne pas à voir, l'inscription corrélative des mots-actes parmi les actes, il ne donne pas d'accès direct à l'effet de sens pratique en situation d'expression du languaging de premier ordre. En situation d'expression de méta-languaging de second ordre, ces effets pratiques occultés doivent être imaginés, reconstruits à partir d'enregistrements mémorisés d'où s'extraient des prédictions par calcul, d'où des effets de sens pragmatiques qui articulent la présupposition et l'anticipation (les implicatures conversationnelles) de manière diffuse ou précise selon les savoirs fournis par le co-texte. Pour cette raison, la pragmatique classique s'intéresse à l'accès aux présuppositions et aux implicatures et envisage la transition du sémantique au pragmatique comme la résolution d'un problème où s'opèrent des calculs en maniant des représentations. Cette démarche fonctionne pour l'expression du méta-languaging de second ordre, mais elle ne s'applique pas au languaging de premier ordre, où la démarche calculatoire, telle que la présente la théorie de la pertinence de Sperber et Wilson 1978 ou la « conversion » dans « la fonction muette du langage » (Coursil 2000), n'est pas requise.

En premier ordre, les agents qui collaborent en catalysant la progression concertée par des actes de languaging, vivent les mots-actes dans le contexte de l'ensemble des actes, et les relient à l'ensemble des savoirs disponibles, immédiats et enregistrés : tout est là, rien n'est à restaurer ni calculer, et les paroles ne font qu'orienter, modifier la conscience de ou l'attention prêtée à une situation, et rectifier la dynamique d'engagement participatif déjà en cours d'exécution et où les rôles sont clairement répartis. Le languaging est imprimé dans chaque mot-acte de manière à la fois générique (savoir à quoi servent les clés d'un camion à partir de discours dans des situations impliquant des clés et des camions) et spécifique (connaître ces clés et ce camion à partir d'un historique dialogique et pragmatique privé), et chaque mot-acte se relie au réseaux d'actes que forme la situation où se joue l'interaction : les phrases-actes sont en prise avec la co-action.

Dans certaines formulations, l'emprise du scénique et du pratique est instituée. Déclarer "Vert », « C'est vert » ou « Le feu est vert » quand on est le passager d'un véhicule, c'est orienter l'attention vers une fata morgana scénique - la couleur du feu - indissociable d'une polarisation pratique - un feu qui passe au vert est une injonction à démarrer. Du fait de l'expérience acquise, des enregistrements de ce type de situation avec ou sans accompagnement de languaging exprimé, l'injonction au démarrage est inhérente à l'idée comme à la formulation du feu vert : sens scénique et pratique ne font qu'un; ils coïncident de manière indifférenciée, même si pour en parler en métadiscours de second ordre, nous ne pouvons que les faire distinguer par les mots dont c'est le rôle. Dans la vie réelle, le conducteur à qui on dit «c'est vert " n'a pas à calculer ce que l'on chercherait à lui faire inférer en lui soumettant cette vision: il s'inscrit directement dans une

Coluche, Le Schmilblick, "... c'était pour dire à Zézette qu'elle aille directement chez René parce que comme j'ai paumé les clés du camion, on va être emmerdés pour lui livrer l'armoire" https://www.youtube.com/watch?v=movRu_SSh_s 
situation connue à laquelle il réagit par un modèle d'ajustement comportemental adéquat pré-enregistré qu'il n'a qu'à reproduire par "réflexe lucide », sous surveillance réflexive. En second ordre, pour un observateur en mode spectatoriel, l'expression " c'est vert » symbolise un modèle de situation stéréotypique ; en premier ordre, pour un expérient en mode actoriel, elle fait énacter un modèle d'engagement dans ce type de situation.

\section{Autres effets de sens}

Puisque l'effet de sens est une distinction réalisée en expression de languaging de premier ou second ordre selon la diversité des engagements participatifs et points de vue observationnels que l'on peut y convoquer, il ne faut pas s'attendre à une typologie réifiée qui contredirait la démarche même, mais à une série de processus configurés générateurs d'effets émergents et complexes (cf. l'herméneutique de Rastier 1996 et 2005).

Un énoncé produit un effet de sens onirique si ses composantes font obstacle à l'élaboration d'un effet scénique cohérent, comme le vers chomskyen ; mais tout est affaire d'intentionnalité : un internaute a réinterprété Colorless green ideas sleep furiously comme commentaire aux débats entre écologistes pendant les primaires, "les idées vertes dorment furieusement »: en simulant cette inscription cruellement plausible, il a produit la distinction d'un sens scénique, très imagé, et d'un sens pratique, la lassitude de l'électeur blasé, et neutralisé l'onirisme de l'énoncé initial, fondé sur deux règles internes d'auto-engendrement, (i) la séquence accentuelle et rythmique 10010101 100, et (ii) la progression lexicale par contradictions et contrastes (colorless vs green, green matériel vs ideas immatériel, ideas spirituel vs sleep d'un corps vivant, sleep inerte vs furiously énergique), ce qui fait de ce vers l'expérience autopoïétique par excellence, le parcours poétique expérimental imaginé par Borges et nommé hron dans la fiction Tlön, Uqbar, Orbis Tertius (1944).

Les énoncés fabriqués par les linguistes en second ordre, comme «le chat mange la souris » entendent produire un effet de sens théorique, de type scénique, mais excluant tout intérêt pour l'inscription bio-écologique en premier ordre, d'où des formulations souvent artificielles, improbables en situation réelle (Goffman 1987, 37). Le sens théorique peut mener à des erreurs d'analyse, comme le caractère prétendument tautologique de «la neige est blanche » (la blancheur étant une propriété inhérente de la neige) : en premier ordre, un énoncé de ce type ne peut être proféré que par un instituteur face à des élèves, ou un parent face à un très jeune enfant, qui considèrent que la relation objet / couleur n'est pas encore enregistrée, ou la maîtrise des mots et accords pas encore acquise, et qu'elle se pratique par des activités de verbalisations pédagogiques et ludiques ; l'effet de tautologie résulte de la décontextualisation, de la neutralisation du sens pratique et d'un intérêt exclusif pour l'analyse logique, comme si le languaging de premier ordre était contraint par la discipline des logiciens ${ }^{13}$. La pratique de discours rhétorique visant à conventionnaliser des protocoles de raisonnement de nature à ce que la concorde s'impose d'elle-même sans passer par les techniques sophistes de séduction subjective par l'émotion imprime a posteriori dans le languaging des enchaînements morphosyntaxiques et stylistiques rituels (connecteurs, corrélations, protases et apodoses dans les phrases conditionnelles) qui lexicalisent et grammaticalisent dans le discours des éléments de raisonnement logique motivés par une idéologie politique et une démarche finalisée où la raison grammaticale de second ordre oriente le devenir du languaging de premier ordre, et joue un rôle acculturant constitutif (Danblon 2013). Les maximes conversationnelles de Grice ne décrivent pas les propriétés pratiques du languaging profane, elles prescrivent des objectifs normatifs à atteindre pour un languaging rendu expert. Inversement, un grammairien comme Bolinger explique une structure anglaise comme I'm happy to see you par

13 Cf. également l'expression tri sélectif, qui n'est tautologique que si on occulte le rôle de sélectif dans la distinction de l'acte générique de tri et de sa finalité sélectionner, très importante pour l'inscription écologique de la notion même, qui coordonne un changement à imprimer à l'environnement. Je monte en haut n'est pas tautologique, haut distingue l'opération du résultat et oriente l'attention vers le lieu où l'on me trouvera si on me cherche, préparant les interactions ultérieures possibles. 
l'illogisme naturel de l'esprit humain, considérant que l'infinitif to inverse la relation de la cause $I$ see you à l'effet I'm happy, comme si to ne pouvait que refléter un enchaînement logique (les théories de l'énonciation ont diverses idées sur la question, cf. Bottineau 2001).

Les locuteurs se vivent souvent comme si leur production verbale faisait sens scénique alors que ce n'est pas le cas du point de vue du linguiste. L'expression on ne peut pas nier le contraire comprend trois négations qui devrait faire produire une scène contraire à l'intention du locuteur, mais celui-ci ne visualise pas le sens : il fait progresser la résolution de la polémique à coup de négations, et ça fonctionne si on ne prête pas attention à la synthèse. A la question "Qu'est-ce que ça boit, une vache ? », beaucoup d'interrogés répondent sans hésiter " Du lait », en raison de l'effet d'amorçage occasionné par la fréquence de co-occurrence des mots vache et lait dans le dialogisme enregistré. Nombre d'expressions comme faire un virage à 360 degrés présentent ce type d'anomalie représentationnelle. L'effet de sens pratique de ces pragmatèmes est si rapide et efficace qu'il provoque l'arrêt immédiat du processus interprétatif et bloque la recherche d'un effet de sens scénique devenu inutile, ou laisse en arrière-plan une scène diffuse dont la connexion avec la forme langagière n'a pas à être traitée. On ne compte pas les structures qui disent autre chose que ce qu'elles suggèrent littéralement, comme tout le monde ne peut pas en dire autant, qui signifie pas tout le monde peut en dire autant (cf. anglais not everyone can say as much); diverses études (dont celles réunies par Mattia-Viviès 2012) s'intéressent à la syntaxe mensongère (deceptive syntax), qui n'apparaît mensongère que si l'on décide en second ordre que la formule doit faire sens en elle-même. De manière générale, comprendre, c'est se satisfaire de la participation de la verbalisation à l'interaction en tant que coordination de coordinations consensuelles récursives, et non pas chercher à élaborer une représentation comme fin en soi. Les discours managériaux, politiques et publicitaires exploitent l'effet de satisfaction apporté par la familiarité des «éléments de langage " en lesquels chacun croit se reconnaître. Si le pragmatique est postérieur à l'épistémique en second ordre, en premier ordre, c'est l'inverse.

\section{Le sens de la syntaxe}

Le terme et la notion de syntaxe, constitués depuis la Grammaire de Priscien (Livre XVII, Syntaxe-I) (Baratin 1979), signifie étymologiquement rangement des mots et recouvre plusieurs démarches de second ordre : (i) la distinction de "parties du discours " (classes de mots) au sein de la phrase écrite par segmentation et inventaire de catégories d'unités selon les critères de placement, associations ordonnées par constructions, et variations morphologiques (cas, temps); (ii) la production de phrases conformes aux règles d'arrangement, en particulier dans la perspective de l'enseignement de la rédaction de discours composés dans le respect de l'art de la persuasion oratoire, la rhétorique. Selon Lallot (1988), la syntaxe émerge par étapes : (i) les scribes Mycéniens (linéaire B) commencent à distinguer les mots écrits en introduisant des espaces séparateurs marqués par un trait vertical ; (ii) Homère voit dans le lexique un inventaire classé des choses du monde ; (iii) le Cratyle platonicien segmente le logos en deux composantes, onoma et rhêma, dont Aristote précise les propriétés ; (iv) les Stoïciens élargissent l'inventaire des classes de mots, que les Alexandrins précisent et complètent, avec notamment l'inventaire de référence de la Tekhne Grammatike de Denys le Thrace ; (v) Apollonios Dyscole élabore une "sémantique » lexicale qui associe des traits sémantiques et des propriétés formelles et combinatoires.

La syntaxe représentée et modélisée en linguistique est typiquement l'abstraction de second ordre qui se développe par observation de la forme écrite, distinction de composantes, de leurs variations formelles et positionnelle, interprétation des hiérarchies et contenus de relations, manipulations analytiques (commutations) et synthétiques (rédaction). Ces dernières atteignant leur climax avec le générativisme, modèle d'auto-production des structures syntaxiques instanciables par le lexique et les morphèmes. Une structure syntaxique est un observable de second ordre produit par une démarche métadiscursive intentionnelle orientée par des finalités spécifiques telles que la mise au 
point de techniques de scription, de rédaction, de persuasion, de description par inventaire et classification, et plus récemment d'explication du phénomène langagier par production de modèle calculatoire. Cette construction occulte l'inscription bio-écologique du languaging de premier ordre. Elle convient pour programmer du languaging automatisé en environnement numérique et allopoïétique, mais pas pour le langage humain, ou du moins : pas sans s'être demandé comment les structures syntaxiques peuvent être réalisées en languaging de premier ordre comme modalité de l'activité productrice de monde vécu et constituées comme observables à ce niveau par les agents réflexifs opérant des distinctions, des commentaires et des manipulations.

\section{La syntaxe comme enchaînement auto-engendré}

Le rapport observationnel à l'écrit fait apparaître une toposyntaxe, à savoir un tout construit dont les segments apparaissent simultanément, synchronisés. A l'oral, l'activité de phonation fait enchaîner des étapes lexicales et morphologiques, et fait apparaître une chronosyntaxe, un enchaînement en devenir dont chaque pas, comme en marche et en danse, s'appuie sur le précédent et prépare le suivant, réalisant une transition au sein d'une locomotion, étymologiquement un "mouvement relatif au lieu ». Le terme chronosyntaxe a été proposé par l'hispaniste Macchi $(2000,2006,2008)$ à la suite de la notion de chréode (Delport 2008) "parcours syntaxique ", " trajet ", avec une séquence de postes à instancier (gabarits, templates pour les grammaires de construction); les chronosyntaxes désignent les syntaxes de second ordre qui modélisent le facteur temps qui sous-tend l'activité de l'agent de languaging en premier ordre, avec trois principes :

(i) à chaque moment de l'enchaînement de parole est introduit un opérateur qui opère des effets de rétroaction (reprofilage d'acquis en mémoire de travail cursive) et de proaction (ouverture d'anticipations prédéterminées par les routines récurrentes, qui coordonnent l'auto-détermination de la poursuite énonciative et l'attente interprétative) : chronosyntaxe de Macchi, on-line syntax (Auer 2009) et grammaire instructionnelle (Col et al. 2012);

(ii) selon le type linguistique, les catégories syntaxiques de second ordre (sujet, objet) sont soit fixées en premier ordre par la position (le moment) ou la forme casuelle (cas du français : pronoms sujet et objet; basque : les postpositions), soit en devenir : une focale initiale devient sujet, objet ou attribut a posteriori, en fonction d'une cascade de rétroactions) (Denez 1986, Bottineau 2016a pour le breton, Blestel (à paraître) pour le guarani ; Bottineau (2011a) pour le basque). Exemple breton : un disglavier a zo ganin 'un parapluie est avec moi' "j'ai un parapluie ", un disglavier am eus prenet 'un parapluie me-est acheté' "j'ai acheté un parapluie ». La focale "parapluie » devient sujet a posteriori, en fonction de ce qui suit, par réinterprétation ultérieure; la catégorisation syntaxique n'est réalisée ni par la position, ni par une marque de cas type nominatif vs accusatif. En premier ordre, le syntagme n'est pas sujet ou objet au moment de sa formulation, il le devient. De même, un groupe nominal sémantiquement incomplet peut être pris comme sujet provisoire avant d'être modifié : breton Per a zo brav he ti 'Pierre est beau sa maison' "Pierre a une belle maison ". Les grammaires de second ordre parlent de sujet disloqué, comme s'il s'agissait d'une transformation déstructurante, alors que personne n’a jamais rien " disloqué » : un sujet qui apparaît disloqué en toposyntaxe de second ordre pour le linguiste spectatoriel est en réalité un sujet en devenir pour un parcours chronosyntaxique de premier ordre réalisé par des interlocuteurs en mode actoriel, entraînés à ce modèle de routinier coordination. Enfin, selon le type linguistique, un couplage lexique / catégorie peut être irrévocable ou réajustable (anglais : the book you sent me is really interesting, où the book reçoit à des moments différents un rôle de sujet en attente de verbe et un rôle rétroactif d'objet du verbe sent qui n'en a pas pour lui-même, avec une intonation et un rythme qui pilote le réseau des connexions en temps réel ; breton : l'objet d'un verbe antérieur peut être repris comme sujet d'un verbe ultérieur sans pronom relatif, avec prédications en série et recatégorisations en temps réel). Du fait des dynamiques d'émergence en premier ordre, les notions de syntagme et de fonction ne sont pas des primitives descriptives adaptées à toutes les langues : derrière des objets linguistiques aux contours instables ou disloqués se cachent des processus et parcours en progression et révision. 
(iii) selon la fréquence de pratique et la relation à la prosodie (continuité du groupe de souffle et du schème intonatif), un enchaînement chronosyntaxique peut être figé, parcouru en trajet direct sans accomplir toutes les possibilités de pro- et rétroaction en parcours " omnibus » qu'une analyse de second ordre suggère : les agents parlants observent les enchaînements et peuvent les énacter dans le détail de l'improvisation ou dans la globalité d'enregistrements préconstruits (cf. infra l'exemple de l'antéposition adjectivale en français) ; les trajets chronosyntaxiques unifiés en premier ordre livrent en second ordre les figements, les constructions, et la non-compositionnalité.

La grammaire de second ordre travaille avec des structures syntaxiques statiques (toposyntaxiques), que les chronosyntaxes transforment en parcours (comme la chréode) : c'est une avancée, mais il faut préciser comment émerge un parcours en languaging de premier ordre sans présupposer la vision d'ensemble d'un trajet muni d'étapes prédéfinies sans improvisation possible. Certains modèles intermédiaires comme De Fornel et Marandin (1996) suggèrent que le locuteur en production orale observe ses produits syntaxiques qu'il complète par auto-réparation.

Par analogie avec la marche, on envisage ici le languaging instantané comme une activité d'auto-locomotion, avec une séquence de transitions d'états instables à états instables, chaque état étant instable du fait de corréler une rétroaction qui récupère en boucle un enregistrement orientateur et une proaction qui coordonne les poursuites énonciative et interprétative en conjoignant l'action et la mémoire de travail (Baddeley 2003). Chaque acte momentané est ainsi corrélé à un avant et un après qui permet d'improviser l'enchaînement sans disposer d'une vue d'ensemble d'un trajet muni d'étapes, exactement comme chaque étourneau règle son vol sur celui des proches voisins sans se représenter la forme clôturée du vol d'étourneaux qu'appréhende l'observateur extérieur (prédateur ou humain). En outre, la règle du languaging du premier ordre est la récursivité et la réflexivité, avec une mise en observation systématique de l'activité «de l'intérieur " par les agents qui participent à la coordination de coordination, ce qui constitue la mise en observabilité des enchaînement récurrents et l'émergence d'enregistrements de parcours syntaxiques de premier ordre du point de vue des sujets parlants, que les méta-observateurs linguistiques de second ordre peuvent modéliser comme des parcours, puis des structures selon le recul pris par rapport à l'inscription biologique. On transite ainsi d'une auto-locomotion (mouvement local relatif aux points d'actes du parleur par couplages de rétroactions et proactions successifs) à une auto-géomotion (progression sur un terrain géographique observé et construit: l'enchaînement syntaxique comme parcours d'un trajet avec étapes, d'une chréode avec postes, d'une construction, avec un parleur muni d'un point de vue).

\section{Le couplage avec la prosodie}

Pour certaines langues, comme le russe, la progression syntaxique est indexée sur des parcours de fluctuation intonative, accentuelle et rythmique : lorsque l'on énonce une phrase en russe, le passage de SN à SV est sensible, scandé par une variation de ton et de rythme, si bien que la structure abstraite de second ordre est réellement ancrée dans l'expérience locomotive de premier ordre. Les parcours syntaxiques du russe sont nettement ancrés dans les variations prosodiques : en premier ordre, les agents font l'expérience de parcours intonosyntaxiques faiblement différenciés que l'analyse de second ordre radicalise en traitant séparément la syntaxe des constituants et les prosodèmes. Cette séparation dénature quelque peu la syntaxe, mais sa désinscription ne se traduit pas par la schématisation de structures abstraites qui n'auraient pas d'existence en premier ordre.

En français, le couplage entre prosodie et syntaxe est plus délicat à établir (Lacheret et Beaugendre 1998, Simon et Christodoulides (2016). Il existe selon Morel et Danon-Boileau (1990) une intonosyntaxe autonome qui gère la progression locomotive du "paragraphe oral " (préambule, rhème et post-rhème) en recrutant progressivement des regroupement lexicaux sans faire coïncider les étapes avec la segmentation de second ordre. Considérons l'exemple Moi, chuis fatigué formé du préambule « moi » et du rhème « chuis fatigué »: le rhème regroupe $\mathrm{SN}$ 'je' + SV 'suis fatigué', d'où la compression je + suis = chuis. Le SN et le SV sont amalgamés, sans production gestuelle et sans observabilité en premier ordre; la question est alors de décider si chuis résulte d'une transformation ou de la réplication directe d'une chaîne dialogique d'exemplaire antérieurs de 
chuis, auquel cas il n'y a pas à s'appuyer sur un modèle d'enchaînement. L'anglais est connu pour ses schèmes prosodiques qui se projettent sur l'énoncé en faisant porter le noyau sur un élément lexical précoce ou tardif, faisant varier complètement la coordination des attentions attentionnelles. Soit ce bref dialogue entre deux militaires extrait du film Dunkirk de Christopher Nolan (2017) :

The tide must be coming in -

- How can you tell? -

- The bodies are coming back -

Dans le contexte, les deux militaires savent que les corps sur la plage ont été emportés par la marée, et qu'ils allaient fatalement revenir. Par ce parcours intonatif, le second attire l'attention du premier sur une apparition présentée comme une révélation attendue : ça allait de soi, tu pouvais remarquer ce détail et l'interpréter sans me poser la question. En français comme en anglais, contrairement au russe, le vecteur prosodique ne chréodise pas l'énonciation, il ne fournit pas de schème de variation rythmique et intonative qui canalise l'exécution d'un enchaînement par phases et étapes préenregistrées. Toutefois, les travaux sur la syntaxe du français parlés issus des approches de Blanche-Benveniste (1990), Kerbrat-Orecchioni (2005), Morel et Danon-Boileau (1990) ont établi qu'en discours oral spontané, avec détachements et redondances (moi, perso, je), les parcours syntaxiques sont solidaires de la prosodie avec des effets de boucle perceptive (Levelt $1989^{14}$ ) et coordinative qui constituent l'auto-locomotion conjointe des partenaires dialogaux. En voici un exemple remarquable issu d'une conversation personnelle : sur un marché, un vendeur rend la monnaie en disant " trois euros je vous reeeeeeends... ? » avec les pièces dans le creux de la main qu'il tend progressivement en accompagnant la durée de "reeeeends ", la montée intonative, et le parcours oculaire qui suit la main et finit par retrouver le contact avec mon regard. Comme il avait reçu un montant problématique (des billets et des centimes pour arrondir la monnaie à rendre), il s'est interrogé sur la somme à rendre, puis a répondu à sa propre question par l'objet initial « trois euros ", accompagnant sa vérification réflexive du geste des mains et du regard, finissant par solliciter la confirmation et l'accord en finalisant le geste, en reprenant le contact oculaire, et en terminant par la montée interrogative. Dans cet exemple, la syntaxe OSV est inintelligible si on ne l'associe pas au geste, au regard et à la voix, et si on ne la motive pas par le travail de coordination requis par la situation involontairement créée. Une grammaire de second ordre sans ancrage au premier ordre ne peut pas expliquer cet «emploi ». Un corpus oral annoté sans enregistrement vidéo qui rende ces enjeux visibles ne permet pas de traiter ce type d'exemple.

\section{Syntaxe et typologie linguistique}

La typologie linguistique est en quête d'universaux de second ordre, comme les universaux implicationnels de Greenberg (1963). De ce fait, il est d'usage de regrouper les langues par types de similarité syntaxique des énoncés transitifs bivalents dans la proposition indépendante (avec groupes nominaux lexicalisés et en faisant abstraction du positionnement des pronoms) : SOV (basque, japonais), VSO (tahitien), VOS (irlandais), SVO (français, anglais), XV2 (allemand, breton). Ces types schématiques apparaissent au prix de manipulation : la production d'énoncés fabriqués ou décontextualisés, l'éviction des pronoms, de la subordination, des modes autres que l'indicatif (en particulier l'impératif), le choix de la transitivité ou de la bivalence comme dénominateur commun universel, et surtout l'idéalisation d'un "schème canonique » qui repose sur l'occultation de l'inscription, des variations d'ordre avec l'intonation, la gestualité. Toutefois, on peut s'appuyer sur cette approche à condition d'expliciter l'orientation des coordinations de premier ordre que les parcours de second ordre révèlent. En basque, l'ordre SVO avec accord multiple final traduit une progression qui consiste à d'abord construire des arguments lexicaux qui se positionnent

14 On retient de Levelt (1989) la notion de boucle et de rétroaction, mais on ne l'interprète pas comme information réentrante : Levelt modélise le phénomène avec des présupposés internalistes et calculatoires incompatibles avec la cognition énactive. 
successivement par les postpositions de relations syntaxiques (par auto-locomotion), avant de terminer par le verbe où se regroupent les indices des principaux actants dans le contexte des repérages de temps et modalité : Nik Joni eskutitza bidali diot 'moi-ergatif, Jean-datif, la lettre-absolutif, envoyé, je-la-lui' $\rightarrow$ « J'ai envoyé la lettre à Jean ». Métaphoriquement, cela revient à préparer un plat en cuisinant les ingrédients requis séparément (moi en rôle d'agent, Jean comme destinataire, la lettre comme patient), et en les regroupant à la fin (diot 'je-la-lui'; ou à jouer une pièce de théâtre en commençant par le dramatis personae (par couplage d'actants et d'acteurs) avant de jouer l'intrigue proprement dite (le groupe verbal avec accords et paramètrages). Même en négligeant la prosodie et l'inscription bio-écologique en générale, la syntaxe de second ordre à elle seule fournit un modèle scénarisé efficace d'acte de coordination compatible avec sa spécification en contexte de languaging de premier ordre multifactoriel. Le français et l'anglais présentent des scénarios différents.

Dans la diversité des langues du monde, on peut modéliser une typologie des modèles de couplages syntaxiques, plus ou moins conjoints à la prosodie selon les cas (le degré d'intégration ou de modularité distinctive étant lui-même une variable typologique et non un choix dogmatique de la théorisation). En outre, l'interprétation du modèle de couplage porté par un schème donné est aussi affaire de distinction et de point de vue : en breton parlé, le syntagme initial est interprété comme foyer d'attention conjointe (Didier eo ma anv 'Didier est mon nom' "Je m'appelle Didier »), comme dans le Yoda speak de Star Wars (Revealed your opinion is!), typique des parlers celtiques des sagas arthuriennes. Ce modèle de couplage est préservé à l'écrit chez les auteurs qui entretiennent le "génie celtique " du breton, mais on voit également apparaître l'interprétation inverse, avec l'élément initial thématique comme en français : Me a zo Didier 'moi est Didier' "Je m'appelle Didier », grammaticalement "correct " mais non conforme à l'usage traditionnel. Ce renversement est lié à l'introduction de l'écrit : à l'oral, l'auto-locomotion oratoire est motivée par la pression écologique de la présence de l'auditeur, qui attend la réponse à la question qu'il se pose, «Didier»; à l'écrit, l'auto-locomotion rédactionnelle est motivée par une autre pression écologique, celle du déjà-écrit que le rédacteur a sous les yeux au moment de poursuivre : la vision des traces externes de sa propre mémoire graphique l'invite à poursuivre du connu vers l'inconnu, de l'ancien vers le neuf, donc du thématique vers le rhématique, conformément à la séquence onoma - rhêma platonicienne issue de l'écrit. En breton, la réinterprétation du modèle de coordination appliqué au schème syntaxique (focale vs thème à l'initiale) commence avec la traduction des textes religieux en breton d'église destiné aux paroissiens bretonnants, et elle s'intensifie avec la propagation de divers bretons académiques à des apprenants du breton langue seconde issus de milieux non brittophones, sous forte influence du français normatif et des pratiques écrites de l'enseignement. La même bascule a été observée par Combettes (2012) pour le français médiéval, avec des effets de cohabitation des deux lectures du même schème dans les mêmes textes, et les effets d'incohérence et de ruptures de cohésion discursive que cela implique. La « raison graphique » a pour conséquence d'incorporer la source de pression écologique de l'auto-motivation rédactionnelle au texte lui-même, ce qui en fait un processus autopoïétique plus radicalement clôturé : le rédacteur d'un texte trouve sa source d'inspiration au sein même des produits de son activité du fait d'être confronté visuellement aux traces écrites de sa propre production, alors que l'improvisation orale se nourrit d'une boucle à l'empan temporel restreint du fait de sa fugacité. En allemand parlé, un locuteur produit une phrase à verbe initial (au lieu de V2) s'il intervient en réponse à une réplique antérieure ressentie comme stimulus du fait des paramètres situés immédiats (intonation, attitude) et culturels (rapports hiérarchiques...) : hab'ich schon gemacht " c'est fait !". Les types syntaxiques de second ordre doivent plus ou moins au divers types d'inscriptions en concurrence selon les traditions de parole et d'écriture qui animent les communautés et leur prise en compte par les rédacteurs de descriptions selon leur ouverture observationnelle et leurs motivations (celles d'un prêtre qui convertit les âmes ne sont pas celles du linguiste qui s'adresse à ses pairs). Une typologie syntaxique qui nivelle les structures en effaçant leur terreau inscriptionnel produit un modèle exposé au risque de contrefaçon. Certaines approches comme Lambrecht (1976) abordent la 
question en termes d'ordre de la chaîne informationnelle, mais elles restent dans le paradigme de l'encodage et la transmission d'une information.

Les sujets parlants observent-ils les structures syntaxiques?

Compte tenu de la rétrospectivité ouverte de l'auto-locomotion et de la constitution de segments observables par la réplication, la récursivité et la réflexivité, les locuteurs tendent à former des objets lexicaux et des gabarits syntaxiques parlés et écrits qu'ils manipulent au sein de l'épi-discours en acte comme des objets symboliques. Ceci donne le est-ce que interrogatif, l'antéposition de l'adjectif avec ses effets d'inscription dialogique (curieuse bête; drôles de gens que ces gens-là), les figements (un tiens vaut mieux que deux tu l'auras), le commentaire et la recatégorisation des pragmatèmes (je suis carrément pas d'accord: pas d'accord est figé en adjectif attribut, cf. carrément méchant), les nominalisations par conversion (a no-go area; good eats to be had in New York), etc. En outre, toute manipulation syntaxique en premier ordre retravaille le profil de la coordination de coordinations, son inscription interactionnelle et écologique, selon divers points de vue en fonction de la tradition de languaging enregistré à laquelle la manipulation s'adosse. Sans se plonger dans la diversité des travaux sur la question, considérons sommairement le cas de l'antéposition de l'adjectif en français :

- la postposition ajoute au nom une spécification de propriété ou de jugement : un vin blanc, un vin délicieux.

- pour un jugement non institué (une demeure somptueuse), l'antéposition présente le jugement comme déjà opéré, préconstruit, offert à l'observation et à la mise en scène (une somptueuse demeure : le locuteur se présente comme commentateur du déjà-dit de lui-même ou d'autrui.

- pour un jugement institué, l'antéposition est de règle du fait de l'ancrage dialogique du jugement : un bon vin (= un vin qu'une chaîne dialogique a déclaré bon). Les adjectifs de jugement institué sont, en français, ceux qui mesurent des propriétés et qualités gradables de première importance pragmatique (grand / petit, bon / mauvais) et de nature à figer des sous-classes comme un bon vin, une grande ville. Un adjectif qui ne démarque pas la mesure de la moyenne se prête mal au jugement institué, qui suppose une convergence sur une saillance (une moyenne ville : attesté, mais ressenti comme maladroit, sauf en contexte corrélatif les petites et moyennes villes). L'antéposition mettant le jugement institué en observation, elle peut aussi impliquer le jugement (une bonne femme n'est pas une femme qui est bonne), dont la teneur est spécifiée en contexte (intonation et regard méprisant: Ah les bonnes femmes!). L'antéposition de l'adjectif est ressentie comme neutre et discrète pour les jugements institués du fait du déterminisme dialogique, alors qu'elle est perçue comme remarquable, intense, emphatique et intentionnelle en l'absence de cette pression. Pour cette raison, il n'est pas possible d'attribuer à l'ordre $\mathrm{Adj}+\mathrm{N}$ un sens représentationnel ou pragmatique invariant : l'effet de la manipulation d'inversion dépend de sa motivation dialogique ou subjective, donc de son inscription interactive. L'auto-locomotion de premier ordre participe à la production d'effets de sens, mais la syntaxe formalisée de second ordre n'est pas intrinsèquement signifiante en tant que module séparable : au contraire, la séparation par désinscription la rend insignifiante, d'où le consensus auto-motivé qui règne sur la question.

\section{Sémantiques lexicales : la question du sens des mots}

Dans cette section, la morphosémantique lexicale fera l'objet de la même approche que celle de la syntaxe: les distinctions figées de second ordre, comme les mots-objets dans la phrase ou le signifiant et le signifié spectatoriels, résultent de distinctions émergentes opérées par les agents de languaging au niveau des mots-actes, en instance d'auto-animation coordinatrice et avec divers gestes et degrés de récursivité et réflexivité co-constituantes d'observateurs et d'observés. Cette approche est inaugurée par la chronosignifiance de Poirier (à paraître), selon laquelle une corrélaction créative fait émerger une corrélation observable. La modélisation des degrés du devenir du mot-action en mot-objet au sein du premier ordre passe par la prise en compte de la 
progression de ce rapport corrélaction / corrélation et des étapes de discernement des unités signifiantes, ou symbolification.

\section{Discerner les mots}

Outre les questions historiques abordées en section 3 (syntaxe), la possibilité de discerner des mots lexicaux ne va pas de soi et constitue une variable typologique importante : plutôt qu'une évidence empirique de départ, le mot est une construction. Les mots des langues à déclinaison varient formellement par les suffixes; les mots des langues celtiques varient à l'initiale par un jeu de mutations consonantiques commandées par les marqueurs grammaticaux qui les introduisent (tad ha mamm "père et mère ", ma zad ha ma vamm "mon père et ma mère "); la voyelle des mots turcs varie par le jeu de l'harmonie vocalique (nivellement de voyelles en séries au sein de groupes de mots) ; les mots de langues agglutinantes (basque, inuktitut) se forment par accrétion d'éléments formateurs et de radicaux (basque betaurrekoak 'les (choses) de devant les yeux' $\rightarrow$ «les lunettes ») qui produisent des unités lexicales convenues ou novatrices: dans ces langues, l'agglutination par des processus conventionnels intègre la néologie comme un processus ordinaire de fabrication lexicale, ce qui rend problématique l'inventaire du lexique par les dictionnaires. Même en français, le mot-acte est très plastique et subit nombre d'ajustements opportunistes par divers processus de phonétique articulatoire, comme l'omission et les assimilations progressives et régressives de divers traits moteurs tels que le voisement ou la palatalisation (j'vais m'couper les ch'feux; le PSG prononcé pé èche gé); il subit également des traitements intentionnels de troncation (anglais perambulator $\rightarrow$ pram «poussette »), apocope (covoiturage $\rightarrow$ covoit'), aphérèse (problème $\rightarrow$ blème), apocope avec suffixation (apéritif $\rightarrow$ apéro), verlanisation (femme $\rightarrow$ meuf, arabe $\rightarrow$ beur $\rightarrow$ reubeu $\rightarrow$ reub), métathèses (infarctus $\rightarrow$ infractus), télescopages (foultitude), substitutions phonémiques $(z u t \rightarrow$ flûte) et bien d'autres - autant d'indices que les mots-actes sont flexibles, observés et retraités par des super-actes eux-mêmes conformes à des modèles de routines : i’n'n'a cor / pu «il y en a encore / il n'y en a plus », l'a un blème çui-là «il a un problème, celui-là ".

Le mot-acte étant réalisé par l'action corporelle de l'agent parlant, ce moment d'existence déterminé par le corps propre est ressenti comme la propriété de l'exécutant qui l'interprète, d'où l'éventail très ouvert des variations possibles. La réitération du mot-acte relie mécaniquement le locuteur à la chaîne dialogique des locuteurs précédents qui ont produit des exemplaires antérieurs en se conformant au même modèle, qui peut être conservateur (femme $\rightarrow$ femme) ou réformiste $($ femme $\rightarrow$ meuf). L'invention d'une forme novatrice par un processus conventionnel affiche un acte de rébellion (curé $\rightarrow$ reuc, verlanisation inattestée à ce jour à ma connaissance ${ }^{15}$ ) et sa reprise, l'adhésion à la communauté rebelle qui institutionnalise la transgression en le répliquant (meuf). La figure lexicale jouée caractérise le positionnement de son acteur en termes conformes ou non conformes relativement à l'intériorité et à l'extériorité de sa communauté d'inscription momentanée, ce qui fait proliférer les effets selon les angles de vue et assimile la création lexicale à une perturbation en milieu écolinguistique (Steffensen 2014): l'individuation se forge dans le courant du collectif (Simondon 1989). Par exemple, l'intonation brutale du français parlé de certaines banlieues, ressentie comme ordinaire du point de vue des jeunes qui l'utilisent, paraît agressive à qui ne la pratique pas, d'où des malentendus et des tensions, chacun ignorant ou rejetant les repères de l'autre; en anglais, certains accents britanniques, sociologiquement non marqués

15 J'ai testé ce néologisme reuc en l'employant devant des collégiens d’une école privée. Ils l'ont rejeté unanimement en arguant (avec leurs propres mots) que (i) ce mot n'existe pas et ne peut pas exister, parce que (ii) on ne peut pas toucher au mot curé, qui relève du sacré, et (iii) un parent n’a pas le droit de verlaniser : la profanation du lexique est réservée aux rebelles et interdite aux grands prêtres du languaging que sont les parents et les enseignants ; les rebelles ne peuvent plus se produire si les farces de l'ordre les parodient. Mon néologisme transgressait toutes les règles implicites de leur domaine consensuel de languaging, qu'ils ont clairement observées en les formulant en situation de crise. 
pour leurs adeptes, paraissent particulièrement "snobs » pour un auditeur Américain qui croit y détecter une affectation intentionnelle. La caractérisation d'une communauté parlante en fonction de pratiques créatives jugées conformes de l'intérieur mais déviantes de l'extérieur a des implications politiques et sociétales sur la manière dont les communautés se perçoivent réflexivement et réciproquement dans une arène d'interactions verbales où se profilent des rivalités de parlance et se gèrent des rapports réciproques de dominant à dominé très conflictuels.

\section{La sémantique lexicale est dialogique}

Le monde produit par le mot-acte en tant qu'unité d'auto-animation coordinatrice restaure les mondes antérieurement produits par les éditions enregistrées du même mot-acte par les partenaires de l'interaction : "Les mots sont de puissantes madeleines " (Robert 2003) ; leur " manducation » (Jousse 2008) s'inscrit dans ses propres contextes cumulés; le sens lexical articule l'expérience verbale et non verbale enregistré en situation et en contexte (Honeste 2011, 2015).

Par définition, le mot-acte réplique et, sans le " s'avoir », cite le même mot-acte de tous les discours de soi et d'autrui où il s'est déjà proféré (Bottineau 2012c, 2013c). Le mot-acte de premier ordre tend à restaurer l'enregistrement d'inscriptions interactionnelles antérieures, et la mise en observation du phylum dialogique dépend de l'attention portée au mot-acte comme segment d'auto-animation muni d'une portée particulière en fonction de ses oppositions avec d'autres. Par exemple, voiture peut être ressenti comme opposé à auto et peut évoquer un registre relativement contrôlé, alors que automobile ne peut pas manquer de paraître archaïsant, comme chaussure vs soulier (qui ne s'entend plus guère que dans le Petit Papa Noël de Tino Rossi); ou que bagnole, caisse, tire et bouse sont spécifiés par des chaînes d'inscriptions dialogiques porteuses d'interactions dialogales très colorées (comme le «alors, tu la gares, ta bouse ? » De Samy Naceri dans le film Taxi de Luc Besson, 1998). Dans les langues romanes, on observe une tendance à démarquer un lexique à filiation dialogique experte avec des racines archaïques (gustatif, vision) d'un lexique populaire à filiation dialogique profane avec des signifiants actuels érodés qui occultent la racine latine ou hellénique (goût, voir), avec une résistance de l'écrit académique (rôle de l'accent circonflexe). En espagnol, le même phénomène concerne les attaques consonantiques : latin clavis $\rightarrow$ espagnol llave (clé de porte) ou clave (clé musicale, architecturale, etc.) ; plenus $\rightarrow$ lleno "plein» / plenario "plénier », et il mène parfois à des homonymies, comme clamare $\rightarrow$ llamar « appeler» $\rightarrow$ llama «il appelle» vs flama $\rightarrow$ llama «flamme ». En anglais, l'histoire des îles Britanniques a connu des vagues de migrations celtiques, romaines, germaniques Angles, Saxons), scandinaves (Vikings), avec vagues antérieures (invasions Vikings en Germanie avant l'arrivée des Angles et Saxons en Britannia, et en Normandie avant la Conquête par Guillaume en 1066), puis des contacts guerriers (Guerre de Cent Ans : langues d'oc et d'oül), impériaux (l'Empire britannique) et culturels (l'Encyclopédie, les Lumières, les révolutions industrielles et scientifiques). De ce fait, l'anglais distingue aujourd'hui massivement des mots-actes qui continuent de produire des mondes qui conservent les caractéristiques d'inscriptions anciennes alors mêmes que les ethnies qui les ont introduites dans les interactions de languaging se sont dissoutes depuis longtemps: reach "atteindre", germanique (allemand reichen), issu de communautés d'agriculteurs et artisans, évoque un geste corporel visant un objet concret (to reach a bottle on a shelf), alors que attain " atteindre », roman, issu des discours religieux, juridiques, philosophiques, politiques, évoque l'idée d'un geste mental visant un objectif abstrait (attain a goal). De ce fait, un locuteur peut se mettre en scène comme artisan du peuple (we are not going to reach our objectives this year) ou comme penseur de l'élite pendant une présentation formalisée au sein d'un conseil d'administration (we are not going to attain our objectives this year) : le contraste des mots-actes reach et attain prolonge des chaînes de languaging diverses, et chaque locuteur peut se les approprier (ou pas) en fonction de sa propre participation aux actes de languaging sociologiquement situé qui assurent la continuité de ces domaines consensuels d'interaction relatifs. Ce modèle de contraste sous-tend tout le lexique anglais (cf. freedom vs liberty, tired vs fatigued, climb vs ascend, huge vs enormous...). Sans avoir entendu d'explication de second ordre ni être capables d'en produire, les locuteurs reconnaissent les interactions verbales qui convoquent les mots-actes par 
lesquels elles se réalisent. Si le mot rendez-vous est neutre en français, l'emprunt anglais rendez-vous, distinct de date (rendez-vous amoureux dans la vie ordinaire) et appointment (rendez-vous administratif), est profondément marqué par la tonalité des œuvres littéraires anglophones qui ont réalisé son introduction, avec le regard sur l'étrangeté de la culture française que cela suppose (cf. le roman Rendez-vous with Rama d'A. C. Clark, 1973 ; la traduction Rendez-vous avec Rama occulte les connotations du titre anglais). Le languaging est typiquement un système social autopoïétique, clôturé et observateur de son organisation (Luhmann 1990, 1995), qui se réalise par l'inscription relationnelle et la mobilisation de ses agents, lesquels savent comment agir sans nécessairement voir et savoir ce qu'ils font: ils ne choisissent pas nécessairement les mots qu'ils emploient, et la question du choix motivé émerge a postériori avec le discernement réflexif de l'effet des mots-actes répliqués. La question est d'abord de savoir ce que le languaging nous fait et nous fait faire comme participants avant que de savoir ce que nous faisons en parlant en tant que sujets consolidés : les agents parlants sont investis et habités par la poursuite de traditions culturelles qui se perpétuent et évoluent au gré des réplications du languaging, en sorte que des modes de pensée inaugurés par une ethnie donnée en un moment de son histoire peuvent se perpétuer après sa dissolution ou à travers d'autres groupes au gré de la dissémination des modèles reproduits. Le concept pré-théorique et controversé de " génie des langues » ne fait pas autre chose que prêter à la langue en tant que système abstrait et structuré l'inflexion de la créativité particulière propre au languaging pratiqué dans une langue donnée par la communauté ou l'élite qui contribue le plus à son profilage (d'où les clichés du français comme langue rationnelle, l'anglais comme langue pragmatique et empirique, l'allemand comme langue de l'émotion...). Si la notion de génie des langues paraît magique et absurde au niveau de la langue comme structure abstraite en analyse de second ordre, elle prend tout son sens si on la remplace par celle de génie du languaging en analyse de premier ordre, avec génie au sens étymologique de créativité, fécondité : on parle alors de la co-évolution d'un système de pratiques langagières et des mondes imaginaires spécifiques qu'il permet de faire énacter par les membres de la communauté engagés dans les actes de parole qui la fédèrent et la distinguent d'autres communautés langagières par des sensibilités détectables, en particulier pour un observateur extérieur. Certaines œuvres littéraires comme Les mots étrangers de Vassily Alexakis explorent la question en donnant aux langues le statut d'esprits venant habiter les personnages qui les apprennent et les parlent, décrivant à leur manière le fonctionnement du languaging : une coordination de coordinations incarnées et récursives, qui sous-tend et oriente le modelage plastique et adaptatif des acteurs qui la réalisent par interactions verbales épisodiques.

Le mot-acte est le sym-bole (fragment à rattacher à un tout) de l'acte de languaging auquel il appartient avec tout l'historique enregistré de ses inscriptions bio- écologiques, dont le discours occulte et polarisant. C'est pourquoi un mot-acte de premier ordre n'est jamais ambigu dans le contexte où il est réalisé : dans appartement à louer (latin locare) et Dieu soit loué (latin laudare), même si les locuteurs ignorent l'altérité étymologique qui fonde l'homonymie de second ordre, les mots-actes produisent des mondes profilés par les enregistrements de leurs inscriptions antérieures, ce qui résout par avance les problèmes de polysémie sans confronter les parleurs à un problème nécessitant une résolution calculée (cas de simplexité). Les locutions figées (aller à pied, mettre sur pied) pré-inscrivent le lexique (pied) dans des segments de discours étendus qui orientent plus précisément le phylum d'inscriptions enregistrées et prédéterminent des mondes à reproduire qui dissolvent la question du sens littéral du mot lui-même : la non-compositionnalité du figement est essentiellement due à sa capacité à renforcer l'enregistrement bio-écologique autre que verbal. Les grammaires de constructions (Goldberg 1995, 2006) présentent l'intérêt majeur de réviser les conceptions saussurienne et guillaumienne du mot lexical comme unité de langue en introduisant la variation de l'empan des segments et canaux enregistrés par les locuteurs dans l'usage (usage-based grammars): même si l'approche reste encodagiste (par correspondances formes-sens), son inscription bio-écologique est renforcée, et sa production empirique très révélatrice de l'opérativité du languaging.

De même, le changement linguistique s'explique par la co-évolution des inscriptions d'un mot-acte et des mondes coordonnés à la production desquels il participe : tabula (latin) participait 
d'interactions impliquant des objets bi-dimensionnels en bois impliqués dans un paradigme d'activités hétérogènes, artisanales, graphiques, artistiques (planches, fragments d'épaves, tablettes, registres, archives, actes, contrats, testaments, peinture, damiers...). Le mot évolue en tabla plus ou moins à l'époque où l'objet devient tri-dimensionnel (sur pieds) avec un paradigme d'activités en partie conservé, en partie novateur. De ce fait, le mot-acte «table » s'inscrit aujourd'hui dans des épisodes de languaging où l'on mobilise des surfaces sans support ou avec (2-D ou 3-D), matérielles ou abstraites, et dans des répertoires d'activités hétérogènes (table de multiplications / de Pythagore, table à repasser, table de mixage, table granitique), et son inscription est souvent diffuse et ne se prête guère à une analyse désignative (cas de l'enfant à qui sa mère demande de « mettre la table » et qui répond "je la mets où ? »). Vouloir reconstruire a posteriori un parcours métaphorique de second ordre qui dériverait des sens abstraits comme la table technique en 2-D de sens concrets avec la table matérielle en 3-D relève d'une erreur d'inventaire historique, du choix arbitraire de la forme table alors qu'elle prolonge tabula et tabla, et de la croyance que le mot-objet désigne, alors que le mot-acte participe de la convocation d'un schème de collaboration enregistré.

\section{La submorphémie lexicale}

L'un des aspects de l'évocation diffuse réside dans la submorphémie lexicale. Les submorphèmes sont des segments de signifiant lexical, souvent des groupes consonantiques, auxquels la linguistique traditionnelle ne reconnaît pas le statut de morphèmes, mais qui de diversent manières sont associés à l'évocation de modèles sensorimoteurs pertinents pour l'accès à l'idée suscitée par le mot pris dans sa globalité. Les études submorphémiques, controversées depuis «l'arbitraire du signe » saussurien, se manifestent à travers une vaste tradition phonosymbolique depuis Cratyle, mais elles connaissent actuellement un essor théorique et empirique conséquent compte tenu de leur reformulation radicale dans le cadre paradigmatique de l'inscription corporelle au signifiant articulé du signifié lexical et de l'explicitation de ses liens à l'approche énactive. En anglais, le lexique dans ses formes une série de matrices consonantiques (les phonesthèmes de Firth 1930, les éléments idéophoniques de Tournier 1985, les marqueurs sublexicaux de Philps 2002, 2006) qui spécifient un modèle d'acte kinésique pertinent: $s w$ - pour le balancement (swoon "se pâmer ", switch " commuter », swim "nager »; cl- pour la préhension (clinch, cling, clench, clasp, clutter), wrpour la torsion (wrist " poignet», wring «tordre / arracher», wrath " rage ») et une centaine d'autres. Ces marqueurs varient en structure avec le type de langue considérée selon l'organisation motrice de la syllabe et du lexique polysyllabique (répartition des chaînes consonantiques en attaque et coda, échelonnement des forces gestuelles par séquences accentuelles et métriques) (Philps 2008 et Bottineau 2014a pour l'anglais, Guiraud 1967 et Nemo 2006 pour le français, Grégoire 2014 pour l'espagnol, Nobile 2008 pour l'italien, Bohas 2006 pour l'arabe) : matrices consonantiques concentrées sur l'attaque en anglais, matrices de traits distribuées sur plusieurs syllabes en arabe, séquences de consonnes permutables en français (cf. table, tableau et plat, plateau). Les modèles kinésiques sont distribués sur l'ensemble des réalisations corporelles (phonation, préhension, locomotion, participation de l'oculo-motricité à la construction de l'espace visuel et en corrélation avec les autres motricités) : sw- de balancement concerne le geste (switch), l'expérience corporelle (swoon), le rapport à l'objet manipulé (swallow " avaler ", swig " gorgée »), le comportement d'agents extérieurs (swallow «hirondelle » pour son vol), etc. Le submorphème inscrit dans le mot-acte un modèle de corrélation kinésique entre l'articulation verbale et la gestualisé non verbale susceptible d'accompagner l'expérience et la perçaction de l'objet ou du processus : il conventionnalise un réseau de motricités verbales et non verbales corrélatives, faisant du mot-acte un geste lié à l'évocation d'autres gestes ; ceci garantit la capacité du mot à coordonner et orienter des mondes vécus pragmatiques, où se profile un accord sur les schèmes de collaborations enregistrées et prévisibles. Une partie du lexique porteur d'un submorphème donné se relie à une racine commune, mais dans le changement linguistique, les locuteurs tendent également à faire apparaître le submorphème par analogie et «bricolage » (cocodrilus $\rightarrow$ crocodile, par analogie avec croquer, avec cr-de croquer, craquer, crunch), au point de rendre l'explication étymologique non pertinente (Smith 2015). 
Les réseaux de submorphèmes sont ancrés dans les interactions verbales et consolidés par les contributions expertes (littéraires et autres) qui distinguent les analogies en appariant les mots dans des environnements confinés, comme l'usage des mots en cl- (clench, clasp, cling, clutter) dans l'Acte IV du Prometheus Unbound de Shelley, souligné par la critique littéraire. Ces corrélations émergent dans le parler populaire dans maintes expressions figées (to go on a spending spree, to come to a standstill, scarce and scant, a treasure trove); les dictionnaires les soulignent involontairement en définissant les mots porteurs de submorphèmes par des quasi-synonymes porteurs des mêmes marqueurs ; et surtout, nombre de rites populaires d'acquisition langagière les mettent en valeur, comme les nursery rhymes et les contes enfantins (dans We're going on a Bear Hunt de Helen Oxenbury, les enfants parcourent des terrains forestiers, marécageux, herbus en s'accompagnant d'onomatopées porteuses des mêmes submorphèmes que le lexique, comme swishy-swashy pour les hautes herbes) : la sensibilité submorphémique n'est pas innée, elle est cultivée par des rituels d'apprentissage et entretenue dans la "langue » (construit linguistique de second ordre) par l'activité du languaging (de premier ordre). Les enfants y accèdent par des biais différents selon leur expérience, pratiques du milieu social, motivations positives ou négatives, et interprètent différemment les pratiques ancrées dans les interactions verbales selon leur mode d'engagement et de participation. De ce fait, le lexique de second ordre modélisé par les linguistes présente un effet de cohérence très poussé mais qui ne reflète pas nécessairement l'expérience qu'en ont les sujets parlants, qui l'appréhendent de manière vicariante. En recensant les contributions expertes, le linguiste recense le modèle théorique idéal ancré dans la somme des interactions verbales, il ne modélise pas la compétence submorphémique personnelle construite par chaque agent. L'analyse statistique de corpus (Drellishak 2006) livre des résultats différents de l'expérimentation psycholinguistique (Bergen 2004), en attendant les résultats de recherches neurophysiologique en cours (Lavissière et Loevenbruck 2016) sur empirie individuelle. Le linguiste explique les propriétés constatées dans la langue extraite des interactions verbales, alors que le psychologue modélise la capture interprétative des propositions ancrées dans les interactions par le sujet participant : les deux démarches se complètent sans se superposer.

\section{La submorphémie grammaticale}

Les submorphèmes sont également présents dans les marqueurs grammaticaux libres et liés (déterminants, prépositions, conjonctions, affixes flexionnels et dérivationnels). Dès 1933, Bloomfield relève en anglais le contraste $w h$ - / th- qui sous-tend des paires comme when / then, where / there, what / that, et que l'on retrouve sur l'ensemble du domaine indo-européen sous diverses formes : I.-E. Reconstruit $* k^{w} / t$, latin te langues romanes $q u-/ t$-, allemand $w-/ d$-, anglais wh-/th-, langue celtiques insulaires du groupe $\mathrm{P} p-/ \varnothing$, etc. Des observations de ce type réapparaissent sporadiquement dans la littérature (Bottineau 2000) mais demeurent anecdotiques du fait de la forte résistance d'une tradition post-saussurienne à l'idée que le signe grammatical le plus abstrait puisse apparaître « iconique » ou «motivé ». L’hispaniste Toussaint (1983) s'appuie sur le guillaumisme et suggère (i) que l'opposition qu-/t- encode l'opposition amémoriel / mémoriel en psychomécanique du langage, (ii) que l'opposition est marquée iconiquement par les phonèmes vélaire $/ \mathrm{k} /$ (occlusion antérieure précoce et prospective dans la cavité buccale du point de vue du sujet parlant) et /t/ (occlusion postérieure tardive et rétrospective): il inaugure une phonosémantique basée sur l'expérience proprioceptive tactile de l'articulation in situ, selon une phénoménologie en première personne de l'acte articulatoire, et récusant la phonologie traditionnelle, qui observe et classe les gestes en troisième personne du point de vue du phonologue comme observateur extérieur. Plus tard, Toussaint (2001) se demande si ce ne sont pas les idées qui miment l'articulation plutôt que l'inverse : en retournant la relation, il inaugure la problématique de l'émergence et de l'inscription corporelle du fait cognitif. Parallèlement et sans connexion au départ, l'angliciste Bottineau (2001) généralise à toute la morphologie grammaticale de l'anglais l'inventaire des microsystèmes submorphémiques ( $w h / t h, u / i / a, s / t$ et bien d'autres) et nomme cognèmes des phonèmes-actes corrélés à des actes de production sémantiques tels que « recherche une entité non mémorisée $(w h)$ ou mémorisée $(t h)$, faire durer $(s)$ ou interrompre $(t)$ un processus, 
conjoindre $(i)$ ou disjoindre $(a)$ deux entités, etc.; les gloses instructionnelles de second ordre modélisent des actes réalisés par l'agent de languaging en premier ordre mais non reçus ni vécus comme instructions (vs la formation des espaces mentaux par instructions : Fauconnier 1984 et 1997, où les interlocuteurs sont co-spectateurs de configurations mentales construites dynamiquement par une chronosyntaxe mais où ne se joue ni emprise bio-écologique, ni co-émergence ; cf. Col 2010). Les morphèmes grammaticaux comme this sont réanalysés comme des complexes opératoires : this $=$ conjoindre $(i)$ une entité mémorisée $(t h)$ à une nomination à réaliser ou impliciter maintenant ( $s$ ) (this book, this), vs that (nomination mémorisée et disjointe). Les mêmes oppositions sont transcatégorielles et se retrouvent dans le verbe et l'adverbe (yes: approbation ouverte; yet : approbation close, d'où concession et réfutation), ce qui va à l'encontre du consensus régnant sur la morphologie distributionnelle et catégorisatrice. Un opérateur comme this participe de l'interaction située et contribue à des effets spatiaux, temporels, déictiques, modaux et autres selon l'inscription bio-écologique (gestes de pointage, cohésion discursive...) et n'est pas en lui-même spatial ou temporel. La structure cognématique des morphèmes grammaticaux en fait des opérateurs-actes d'auto-animation qui corrèlent des gestes phonatoires à des gestes contextuels qui concourent à faire advenir des mondes conjoints qui solidarisent l'expérience partagée de la diction et celle de l'inscription. Le «sens » du cognème n'est pas dans ce qu'il représenterait ou désignerait mais dans sa contribution participative à ce concours.

La cognématique est diversement représentée dans toutes les familles de langues naturelles, avec un noyau commun comme les couple $i / a$ et $k / t$ fortement lié à des contraintes biomécaniques communes, et d'autres systèmes plus vicariants. Certains phyla de languaging sont très conservateurs, avec la conservation de $k / t$ de l'indo-européen à aujourd'hui dans la romania, alors que d'autres renégocient les systèmes ( $w / d$ germanique), ce qui suppose l'observation intérieure du système par ses agents et le reprofilage du domaine par les participations. En la matière, l'analogie joue un rôle important, avec des émergences parallèles comme in / -ing en anglais et en / -ant en français, qui assimilent les «idées » d'intériorité spatiale et de transitionnalité temporelle dans des domaines grammaticaux différents (flexions verbales, prépositions, pronoms), alors même que ces quatre marqueurs ont des origines disparates que rien ne prédestine à s'harmoniser de la sorte : à travers la progression réflexive des pratiques, le "système " tend à engendrer des cohérences innovantes en diachronie. Pour l'espagnol, une communauté de linguistes, poursuivant le projet d'une linguistique du signifiant (Launay 1986, Molho 1988), revoit actuellement l'histoire des langues ibériques en profondeur à l'aune de ces principes (Macchi 2008, Grégoire 2012, Blestel 2017, Le Tallec 2017 à paraître, Pagès 2017, Fortineau-Brémond et Blestel à paraître, Poirier à paraître b).

La submorphologie est l'étude de l'émergence, de l'évolution et de la participation de ces petits opérateurs d'auto-animation phonatoire dans leur articulation et participation à l'auto-animation générale aux échelles micro- (languaging de premier ordre) et macro- (languaging polarisant). Cet axe de recherche qui sonde "la dialectique perplexe du signe et de l'objet » (Neyrod 2017) peut sembler anecdotique de l'extérieur de la linguistique, mais il est à l'origine de l'intérêt actuel des morphosémantiques lexicale et grammaticale pour l'expérience des opérateurs-actes situés du premier ordre, du réexamen des paradigmes de second ordre, puis de l'importation du regard énactivisant à partir de 2006-2008 avec les écoles thématiques de l'ARCo. Le signe linguistique associe un signifiant de production motrice, l'articulation, à un signifié de production également motrice du fait de la perçaction, ce qui réduit l'altérité de ses facettes indissociables : signifiant et signifié de premier ordre sont de même nature. Les choses du monde de l'expérience vécue sont énactées par des réseaux de schèmes moteurs auxquels le mot langagier vient ajouter un schème phonatoire supplémentaire, modifiant l'ensemble par l'inscription aux chaînes dialogiques du languaging : le mot fait partie des systèmes d'affordances relatifs aux entités ; le mot-acte est vécu comme une propriété kinésique de la chose elle-même énactée comme complexe kinésique hétérogène, non verbal et verbal. L’idée (ou la vision) postérieure d'un mot détaché des entités, puis porteur d'un rapport signifiant / signifié étranger au référent, émerge d'une cascades d'observations distinctrices de divers ordres. Une révision véritablement émergentiste et énactive de la question du 
signe - et du langage en général - suppose que l'on parte de l'expérience des actions constituantes du point de vue actoriel des agents opérateurs qui les accomplissent avant que de s'interroger sur le couplage entre entités distinguées, comme le font les phénoménologies saussurienne et husserlienne, essentiellement spectatorielles sur ce point : à la différence de la linguistique cognitive (Bottineau 2010, Guignard 2011), on place la corporéité au cœur de tout signifiant verbal ou non verbal dans une perspective résolument pragmatiste (Roy 2013), du moins en premier ordre de constitution.

\section{Conclusion}

Dans cette étude, on a opté pour un énactivisme qui ne se pose pas en externalisme à opposer à l'internalisme, ni en interactionnisme à opposer au subjecto-centrisme, mais pour un énactivisme qui se pose en émergentisme radical au sein duquel se discrétisent tous les niveaux ultérieurs, constitutifs des couplages ressentis et observés, conformément à la racine merleau-pontyenne de sa définition ; et ce positionnement oriente toute une conception du languaging et de ses composantes. Le courant du languaging est porteur d'une co-évolution, celle des auto-animations verbales (lexico-grammaticales et prosodiques) en conjonction avec les auto-animations générales (l'action conjointe et concertée et ses effets sur le discernement d'un couplage sujet / environnement) qui concourent à faire advenir et devenir des mondes vécus conjoints et concertés, dont les communautés corporelles et culturelles des agents fédérées et distinguées par des domaines consensuels de languaging vicariants, les langues. Une co-évolution linguistiquement assistée et accélérée se nomme « histoire », terme qui désigne à la fois l'évolution polarisée par le languaging humain et le récit réflexif d'elle-même se faisant discerner des humains par sa propre activité récursive et distinction du tout du système social à partir de la contribution verbale des agents. La co-évolution générale se fragmente (se babélise) en communautés langagières diverses, avec leurs modes d'auto-production propre, incarnés (voix, postures, attitudes : Bottineau 2013a) et énactés : les langues se concentrent sur certaines distinctions plutôt que d'autres, et différencient les mondes communs qu'elles polarisent malgré un réalignement et une convergence continus sous l'effet de la traduction, de la polyglossie, des contacts, des impérialismes créateurs de linguce francce. Le sens linguistique réside dans les mondes que font émerger l'acteur et l'observateur collectifs et agentifs (participant à une communauté) en se concentrant sur une composante ou un échantillon discernés à partir de points d'acte et de point de vue délimités dans un ordre donné, selon un acte donné avec sa procédure intentionnelle, et à un niveau de récursivité et réflexivité donné. Le temps n'est pas prédonné (Buser et Debru 2011), voire n'existe pas (Rovelli 2014), il se produit (Pöppel 1998) en fonction du languaging, à l'instar de la mémoire en tant qu'idée d'elle-même (Rosenfield 1994).

Ce principe émergentiste concerne les plus petits échantillons (les submorphèmes dans une chanson enfantine) comme les plus étendus (les clichés sur le "génie » créatif des langues selon les points de vue intra- et extra-communautaires, les regards sur les appariements d'apparences incarnées et de mentalités reconstruites). Parler, c'est projeter des paradigmes dialogiques ignorés sur des syntagmes dialogaux : en premier ordre, la parole est, comme la fonction poétique selon Jakobson (1963), créative. Le sens linguistique émerge lorsque le languaging de tous ordres semble produire des idées qui se connaissent en tant qu'idées et relativement à des mondes vécus en tant qu'idées qui s'ignorent, mais se relient aux idées langagières par la référence. Le languaging, par sa créativité, induit à la fois sa propre mise en ordre et en désordre (vs Swenson 1992), sa babélisation procédurale et idéative, double tendance que les observateurs amplifient en second ordre et corrigent en faisant dialoguer ou guerroyer les courants divergents. Les agents de languaging de premier ordre opèrent dans un cadre qu'ils savent contraint dans l'exécution tout en ignorant les effets de polarisation du languaging de second ordre, ce qui les amène à gérer dialectiquement la relation de conformisme et de liberté dans l'action immédiate tout en n'ayant qu'une vue très partielle des résultats dans l'expérience, le «sens » dépassant largement ce qu'ils ont cru vouloir faire : avec la créativité régulée et son effet reconditionnant sur le languaging de second ordre, plus 
on parle et plus on retravaille, "à l'insu de notre plein gré », le champ matriciel qui emporte nos verbalisations et "conceptualisations" ultérieures en fixant des repères phraséologiques qui s'ignorent, des "éléments de langage " agissant comme des idéologies auto-générées. Bottineau (2012a, 2013a) définit le langage humain comme une " technique de conceptualisation ». A présent, on précise la formule en distinguant la conceptualisation réflexive de premier ordre (l'idée que l'on se fait du sens des phrases-actes en les discernant et observant) et la conceptualisation non-réflexive de second ordre (l'émergence de mentalités partagées à travers l'alimentation de la matrice par les contributions distribuées): le languaging est une technique de conceptualisation courante. Le languaging agit collectivement et sur lui-même comme une discipline technique d'auto-animation à effets représentationnels émergents de premier et de second ordre ; il hérite de l'autonomie que Baddeley (2016) réserve aux organismes vivants. Le second ordre tend à faire émerger du système de pratiques des domaines idéologiques en partie de source non subjective, tels que les courants politiques, économiques, sociaux, religieux : l'autonomie de la matrice tend à supplanter celle des agents qui l'alimentent, à la manière des fourmilières qui asservissent l'allopoïèse des agents à l'autopoïèse de la collectivité, ou des organismes qui stabilisent des colonies de micro-organismes en cellules interdépendantes. L'auto-détermination en accélération exponentielle de la co-évolution espèce / environnement par et dans le languaging, y compris de l'auto-organisation du languaging lui-même, a des conséquences encore opaques, mais dont certains effets émergent à l'observation, comme les débats sur l'ère anthropocène et l'emballement de l'effondrement de la biosphère, le transhumanisme, l'émergence d'une technosphère, le languaging auto-organisé des automates informatiques en réseau, la nécessité d'une cybernétique de troisième ordre (Kenny 2009), et la perspective d'une éventuelle rupture de couplage annoncée. La représentation n'est pas le point de départ (Harvey 2015), l'effet de représentation est le point de fuite.

\section{Références}

Aristote (2000). Métaphysique (Traduction du grec ancien par J. Tricot). Paris, Vrin.

Auchlin, A. (1995). Le bonheur conversationnel : émotion et cognition dans le discours et l'analyse $\mathrm{du}$ discours. In $\mathrm{D}$. Véronique et $\mathrm{R}$. Vion (eds). Modèles de l'interaction verbale. Aix-en-Provence, Publications de l'université de Provence, 224-233.

Auchlin, A., Filliettaz, L., Grobet, A. et Simon, A. C. (2004). (En)action, expérienciation du discours et prosodie. Cahiers de linguistique française 26, 217-249.

Auer, P. (2009). On-Line Syntax : Thoughtson the Temporality of Spoken Language. Language Sciences, 31/1, 1-13.

Bachelard, G. (1948). La terre et les rêveries de la volonté : essai sur l'imagination des forces. Paris, J. Corti.

Baddeley, A. (2003). Working memory: looking back and looking forward. Nature Reviews Neuroscience, 4(10). 829-839.

Bakhtine, M. (1970). La poétique de Dostoïevki. Paris, Seuil.

Bakhtine, M. (1978). Esthétique et théorie du roman. Paris, Gallimard.

Barandiaran, X. E. (2016). Autonomy and Enactivism. Towards a theory of sensorimotor autonomous agency. Topoi, disponible à http://cepa.info/2471.

Baratin, M. Priscien et la constitution d'une syntaxe latine : Recherches sur le livre XVII des Institutions Grammaticales. In L'Information Grammaticale, 3, 33-37.

Barberis, J.-M. (1992). Onomatopée, interjection: un défi pour la grammaire?, L'information grammaticale 53, 52-57.

Barberis, J.-M. (1995). L'interjection: de l'affect à la parade, et retour, Faits de langues 6, 93-104.

Barnabé, A. (2017). Perspective taking in language: when bodily involvement impacts motion events' descriptions. Langage et énaction: problématiques, approches linguistiques et interdisciplinaires, Signifiances (Signifying), 1, 2, 33-54.

http://revues.clermont-universite.fr/index.php/Signifiances/issue/view/20 
Barrett, N.F. (2016). What is at stake in the disagreement between interactivity and enaction? Constructivist Foundations, 11:2, 249-251.

Bergen, B. K., (2004). The psychological reality of phonæsthemes. Language 80 (2), 290-311.

Bergounioux, G. (1995). Saussure et les représentations de la pensée. In M. Arrivé et C. Normand, (eds.). Saussure aujourd'hui, Linx (nº spécial), 173-186.

Berrendonner, A., Le Guern, M., Puech G. (1983). Principes de grammaire polylectale, Lyon, PUL.

Berthoz, A. (1997). Le sens du mouvement. Paris, Odile Jacob.

Berthoz, A. (2009). La Simplexité. Paris, Odile Jacob.

Berthoz, A. (2013). La Vicariance. Paris, Odile Jacob..

Berthoz, A. et Andrieu, B. (2011). Le Corps en Acte. Centenaire de Merleau-Ponty. Nancy, Presses universitaires de Nancy.

Blanche-Benveniste, C. (dir.) (1990). Le français parlé. Paris, Editions du CNRS.

Blestel, É. (2017). Ko, ningo, luego: an enactive approach to the emergence of an epistemic subsystem in jopara. Langage et énaction: problématiques, approches linguistiques et interdisciplinaires, Signifiances (Signifying), 1, 3, 25-40.

http://revues.clermont-universite.fr/index.php/Signifiances/issue/view/20

Blestel, E. (à paraître). Chronosyntaxe comparée des prédicats verbaux en guarani et en espagnol: pour une autre analyse de la conjugaison. In C. Fortineau et E. Blestel (éds.). Le signifiant sens dessus dessous. Submorphémie et chrono-analyse en linguistique hispanique. Limoges, Lambert-Lucas.

Bloomfield, L. (1933). Language. New York, Henry Holt.

Bohas, G. (2006). The organization of the lexicon in Arabic and other semitic langages. In S. Boudelaa (éd). Perspectives on Arabic Linguistics XVI, Papers from the Sixteenth Annual Symposium on Arabic Linguistics, Cambridge, March, 2002. Philadelphia, John Benjamins, $1-37$.

Bondì, A. (2015). Pour une anthropologie sémiotique et phénoménologique. Le sujet de la parole entre cognition sociale et valeurs sémiolinguistiques. Intellectica, 2015/1, 63, 125-148.

Bondì, A. (2016). Altérité de la parole et socialité du sens : énonciation et perception d'autrui. In Colas-Blaise M., Perrin L. et Tore G.-M. (éds). L'énonciation aujourd'hui, un concept clé des sciences du langage. Limoges, Lambert-Lucas.

Bondì, A. (2017). Entre énaction, perception sémiotique et socialité du sens : phénoménologie de la parole et de l'activité de langage. Langage et énaction: problématiques, approches linguistiques et interdisciplinaires, Signifiances (Signifying), 1, 2, 5-19.

http://revues.clermont-universite.fr/index.php/Signifiances/issue/view/20

Bota, C. (2012). Eugenio Coseriu et le potentiel épistémologique de l'"energeia" langagière. Energeia, 4, 32-48.

Bottineau, D. (2001). To entre l'aspect, l'actance et la modalité. In P. de Carvalho, N. Quayle, L. Rosier, et O. Soutet (éds). La psychomécanique aujourd'hui, Actes du 8 e Colloque international de psychomécanique du langage. Seyssel. Paris, Champion, 49-84.

Bottineau, D. (2010a). La submorphologie grammaticale en espagnol et la théorie des cognèmes. In G. Le Tallec-Lloret (dir.). Vues et contrevues. Actes du XIIe Colloque international de Linguistique ibéro-romane. Université de Haute Bretagne - Rennes 2. Limoges, Lambert Lucas, 19-40.

Bottineau, D. (2010b). Language and enaction. In J. Stewart, O., Gapenne, E., Di Paolo (eds). Enaction: toward a new paradigm for cognitive science. Cambridge, MA, MIT, 267-306.

Bottineau, D. (2011a). La syntaxe phrastique actualise-t-elle l'incidence lexicale ? Etude comparée $\mathrm{du}$ français et du basque. In M. Arabyan, J. Bres, D. Van Raemdonck, T. Ponchon, R. Tremblay, P. Vachon-L’Heureux (éds.). Le Concept d'actualisation en psychomécanique, actes du XIIe Colloque international de l'Association internationale de psychomécanique du langage (AIPL). Bruxelles, 18-20 juin 2009. Limoges, Lambert-Lucas, 103-114.

Bottineau, D. (2011b). Parole, corporéité, individu et société : l'embodiment entre le représentationnalisme et la cognition incarnée, distribuée, biosémiotique et enactive dans les 
linguistiques cognitives. In J.-B. Guignard (dir). Linguistique cognitive : une exploration critique, Intellectica, 56, 187-220.

Bottineau, D. (2012a). La parole comme technique cognitive incarnée et sociale. La tribune internationale des langues vivantes, 52-53, 44-55.

Bottineau, D. (2012b). Le langage représente-t-il ou transfigure-t-il le perçu ? In Lautel-Ribstein, F. (éd.). Formes sémantiques, langages et interprétations : Hommage à Pierre Cadiot. La TILV (La Tribune Internationale des Langues Vivantes). $n^{\circ}$ spécial. Perros-Guirec, Anagrammes, 73-82.

Bottineau, D. (2012c). Profondeur dialogique et morphosémantique lexicale et grammaticale. In L. Begioni et C. Bracquenier (dir.). Sémantiques et lexicologie des langues d'Europe - Théories, méthodes, applications. Rennes, Presses Universitaires de Rennes, 233-257.

Bottineau, D. (2012d). La fabrique de la langue, fabrique de l'humain. In K. Nassikas, E. Prak-Derrington, C. Rossi (eds). Fabriques de la langue. Paris, Presses Universitaires de France, 161-197.

Bottineau, D. (2012e). Le décentrage intersubjectif des marqueurs de spatialité. In J. Bacha et R. Bret-Vitoz (eds). Espace et énonciation, Linguistique et littératures françaises et francophones. Tunis, Les Editions Sahar, 99-107.

Bottineau, D. (2012f). Syntaxe et interlocution en français et en breton. In C. Douay et D. Roulland (dir.). L'interlocution comme paramètre. Rennes, Presses Universitaires de Rennes, 183-206.

Bottineau, D. (2013a). Pour une approche énactive de la parole dans les langues. In G. Louÿs et D. Leeman (dir.). Le vécu corporel dans la pratique d'une langue, Langages, 192, 2013/4, 11-27.

Bottineau, D. (2013b). L’inscription corporelle de la socialité : la linguistique de Maurice Toussaint, une étape décisive vers la linguistique enactive. Cuadernos de Filología Francesa, 24, Hommage à Maurice Toussaint. Cáceres : Universidad de Extremadura, 79-99.

Bottineau, D. (2013c). Remembering Voices Past: Languaging as an embodied interactive cognitive technique. In E.I. Pivovar (ed.). Gumanitarniye chteniya RGGU - 2012: Teoriya $i$ metodoligiya gumanitarnogo znaniya: Sbornik materialov [Readings in Humanities RSUH 2012: Theory and Methodology of Humanitarian Knowledge: Conference Proceedings]. Moscow: RGGU [Russian State University for the Humanities], 194-219.

Bottineau, D. (2013d). OUPS ! Les émotimots, les petits mots des émotions : des acteurs majeurs de la cognition verbale interactive. In N. Chatar (dir.). L'expression verbale des émotions, Langue française, 180, 4/2013, 99-112.

Bottineau, D. (2014a). Explorer l'iconicité des signifiants lexicaux et grammaticaux en langue française dans une perspective contrastive (anglais, arabe). In L. Nobile (éd.). Formes de l'iconicité en langue française. Vers une linguistique analogique (Tome 2), Le français moderne, 82/2, 243-270.

Bottineau, D. (2014b). Grammaire enactive et didactique du FLE. In C. Martinot et A. Pégaz Paquet (éds.), Innovations didactiques en français langue étrangère. Paris, CRL, 185-200.

Bottineau, D. (2015). Les langues naturelles, objets complexes, systèmes simplexes : le cas du basque. In L. Begioni et P. Placella (dir.). Problématiques de langues romanes, Linguistique, politique des langues, didactique, culture, Hommages à Alvaro Rocchetti. Linguistica 69, Fasano : Schena Editore, 55-85.

Bottineau, D. (2016). Linguistique incarnée et énactivisme : quelles collaborations possibles avec les neurosciences ? In A. Rabatel, M. Temmar et J.-M. Leblanc (éds). Sciences du langage et neurosciences. Actes du colloque ASL 2015. Limoges, Lambert-Lucas, 211-232.

Bottineau, D. (2016a). Subordination et prédication sérielle en breton. In H. Bat-Zeev Shyldkrot, A. Bertin et O. Soutet (dir). Subordonnants et subordination à travers les langues. Paris, Champion, 113-133.

Bottineau, D. (2016b). Le languaging (langagement) et l'incorporation de la culture par la langue. In E. Höhn et P. Poliak (eds.). Cudzie jazyky a kultúry v teórii a praxi, Filozofická fakulta Univerzity Mateja Bela v Banskej Bystrici [Foreign Languages and Cultures in Theory and Practice]. Banská Bystrica, Faculty of Arts, Matej Bel University, 197-222. 
Bottineau, D. (2016c). Les particularités du français calédonien (lexique, morphosyntaxe) et leurs enjeux sémantiques, pragmatiques et cognitifs. In C. Pauleau (éd.). Le français calédonien (Nouvelle-Calédonie). Une 'variété régionale' de français au sein de la francophonie. Confrontation de données calédoniennes, américaines, réunionnaises, mauriciennes, hexagonales, Langages, 203, 3/2016, 49-69.

Bottineau, D. (2017a). Incarnation langagière et grammaire des langues naturelles : vers la fin d'un clivage. In J. Dokic et D. Perrin (dir.). La cognition incarnée. Paris, Vrin, 251-294.

Bottineau, D. (2017b). Langagement (languaging), langage et énaction, a tale of two schools of scholars: un dialogue entre biologie et linguistique en construction. Langage et énaction : problématiques, approches linguistiques et interdisciplinaires, Signifiances (Signifying), 1, 1, 11-38.

http://revues.clermont-universite.fr/index.php/Signifiances/issue/view/20

Bottineau, D. (à paraître). Cognématique et chronosyntaxe: la construction submorphémique $s t+n t / d$. In C. Fortineau et E. Blestel (éds.). Le signifiant sens dessus dessous. Submorphémie et chrono-analyse en linguistique hispanique. Limoges, Lambert-Lucas.

Bottineau, D. et Berthoz, A. (2015). Simplex systems of embodied coordination and interactivity. Draft of a paper used in presentations at the symposium Simplexity: Implications for Language, University of Southern Denmark, Odense, March 19-20.

Bourgine P.; Stewart J. (2004). Autopoiesis and Cognition. Artificial Life 10, 327-345.

Brassac, C. et Stewart, J. (1996). Le sens dans les processus interlocutoires, un observé ou un co-construit? In J.-L. Dessalles (éd.). Du collectif au social. Actes des journées de Rochebrune. Paris, ENST, 85-94.

Bravo, F. (2011). Anagrammes. Sur une hypothèse de Ferdinand de Saussure. Limoges, Lambert-Lucas.

Bres, J., Haillet, P.-P., Mellet, S., Nølke, H. et Rosier, L. (2005). Dialogisme et polyphonie Approches linguistiques. Louvain-la-Neuve, De Boeck Supérieur.

Buridant, C. (2006). L’interjection : jeux et enjeux. Langages 161/1, 3-9.

Buser, P. et Debru, C. (2011). Le temps, instant et durée. De la philosophie aux neurosciences. Paris, Odile Jacob.

Cadiot, P. et Visetti, Y.-M. (2001). Pour une théorie des formes sémantiques, motifs, profils, thèmes. Paris, PUF.

Clark, A. (1998). Being there: Putting brain, body, and world together again. Cambridge, MA, MIT Press.

Col G. (2010). Correspondance et mixage d'espaces mentaux dans la construction dynamique du sens. Mémoires de la Société de Linguistique de Paris, 18. Leuven, Peeters, 53-73.

Col, G., Aptekman, J., Girault, S. et Poibeau, T. (2012). Gestalt compositionality and instruction-based meaning construction. Cognitive Processing, 13/2, 151-170.

Combettes, B. (2012). De quelques problèmes spécifiques à l'élaboration d'une grammaire historique. Langue française, 176, 2012/4, 69-83.

Coseriu, E. (1967/2007). Il fenomeno del linguaggio e la comprensione dell'esistenza de l'uomo attuale. In: C. Bota, M. Schiavi (éds.) avec la collaboration et les traductions de G. Di Salvatore, Il linguaggio e l'uomo attuale. Saggi di filosofia del linguaggio. Verona, Edizioni Fondazione Centrostudi Campostrini, 71-96.

Coseriu, E. (1968/2001). L'homme et son langage. In H. Dupuy-Engelhardt, J.-P. Durafour et F. Rastier (éds.), L'Homme et son langage. Louvain - Paris, Peeters, 13-30.

Cosnier, J. (1986). La parole, régulateur émotionnel de la vie quotidienne. In J. Guillaumin (éd). Ordre et désordre de la parole. Bron, Publications du C.R.I. (Centre de recherche sur les inadaptations), 1-5.

Cosnier, J. (1987). Expression et régulation des émotions dans les interactions de la vie quotidienne. Colloque La psychologie sociale et les émotions. Paris, M.S.H., 1-19.

Coursil, J. (2000). La fonction muette du langage. Matoury, Guyane Française, Ibis Rouge Éditions, Presses Universitaires Créoles. 
Cowley, S. (2007a). Cognitive dynamics and distributed language. Language Sciences 29(5), $575-583$.

Cowley, S. (2007b). How human infants deal with symbol grounding. Interaction Studies 8:1, 83-104.

Cowley, S. (2009). Distributed language and dynamics. Pragmatics and Cognition 17(3), 495-507.

Cowley, S. J. (2011a). Taking a Language Stance. Ecological Psychology, 23, 3, 185-209.

Cowley, S. J. (dir). (2011b). Distributed Language. Amsterdam, John Benjamins.

Cowley, S. J. (2013). Naturalizing language: human appraisal and (quasi) technology. AI \& Soc 28, 443-453.

Cowley, S. J. (2014). Linguistic embodiment and verbal constraints : human cognition and the scales of time. Frontiers in Psychology, 5, 1085.

Cowley, S. J. (2016). Biological simplexity and linguistic cognition. Chinese Semiotic Studies, 12/1, 67-91.

Cowley, S. J. (2017). Changing the idea of language: Nigel Love's perspective. Language Sciences 61, May 2017, 43-55.

Croft, W. (2009). Toward a social cognitive linguistics. In V. Evans \& S. Pourcel (éds), New Directions in Cognitive Linguistics. Amsterdam, John Benjamins, 395-420.

Cuffari, E. C., Di Paolo, E., and De Jaegher, H. (2014). From participatory sense-making to language: there and back again. Phenomenol. Cogn. Sci.14/4, 1089-1125.

Culioli, A. (1982). Rôle des représentations métalinguistiques en syntaxe. Paris, Département de recherche linguistique, Université Paris VII, Coll. ERA 652. Réédité dans :

Culioli, A. (1990). Pour une linguistique de l'Énonciation - Opérations et représentations (Tome 1). Ophrys, coll. l'homme dans la langue.

Depraz, N., Varela, F. J. et Vermersch, P. (2001). A l'épreuve de l'expérience. Pour une pratique phénoménologique. Buscarest, Zeta books.

Danblon, E. (2013). L'Homme rhétorique, culture, raison, action. Paris, Cerf.

De Fornel, M. et Marandin, J.-M. (1996). L'analyse grammaticale des auto-réparations. Le Gré des Langues 10, 8-68.

De Jaegher, H. et Di Paolo, E. (2007). Participatory sense-making: An enactive approach to social cognition. Phenomenology and the Cognitive Sciences, 6 (4), 485-507.

Delport, M.-F. (2008). Une linguistique du signifiant ?, Chréode 1, Vers une linguistique du signifiant. Paris, Editions Hispaniques, 11-35.

Denez, P. (1986). Contraintes formelles de la structuration de la phrase bretonne. In P. Carrer et alii. Permanence de la langue bretonne, de la linguistique à la psychanalyse. Rennes, Institut Culturel de Bretagne / Skol Uhel ar Vro, 19-44.

Desagulier, G. (2011). Le programme socio-pragmatique des grammaires de constructions : bilan et perspectives. Intellectica, 2011/2, 56, 99-123.

Douay, C. et Roulland D. (2014). Théorie de la relation interlocutive. Sens, signe, réplication. Limoges, Éditions Lambert Lucas.

Drellishak, S. (2007). Statistical Techniques for Detecting and Validating Phonesthemes. Conferencia dictada en LSA Annual Meeting, Anaheim, CA.

Elimam, A. (2017). Les soubassements énactifs et glottomoteurs de la sémiomorphose lexicale. Langage et énaction: problématiques, approches linguistiques et interdisciplinaires, Signifiances (Signifying), 1, 1, (61-75).

http://revues.clermont-universite.fr/index.php/Signifiances/issue/view/20

Erard, Y. (1998). De l'énonciation à l'enaction. L'inscription corporelle de la langue. Cahiers de l'Institut de Linguistique et des Sciences du Langage, $n^{\circ} 11$, Mélanges offerts en hommage à Mortéza Mahmoudian, tome I, 91-122.

Esteves-Vasconcellos, M. (2015). The New General Systems Theory: from Autopoietic Systems to Social Systems. Revolução eBook.

Fauconnier, G. (1984). Espaces mentaux. Aspects de la construction du sens dans les langues naturelles. Paris, Minuit. 
Fauconnier, G. (1997a). Mappings in Thought and Language. Cambridge, Cambridge University Press.

Firth, J. R. (1930). Speech. London, Ernest Benn.

Fortineau-Brémond, C. et Blestel, É. (à paraître). Le signifiant sens dessus dessous : submorphémie et chrono-analyse en linguistique hispanique. Limoges, Lambert-Lucas.

Frath, P. (2005). Pour une sémantique de la dénomination et de la référence. In Adolfo Murguia (dir.). Sens et références, Sinn und Referenz. Tübingen, Günther Narr Verlag, 121-148.

Frei, Henri. (2011) [1929]. La grammaire des fautes. Rennes, Presses Universitaires de Rennes.

Frith, C. (2007). Making Up the Mind, How the Brain Creates Our Mental World. USA, Malden (MA), Blackwell.

Froese, T. (2012). From adaptive behavior to human cognition: a review of Enaction. Adapt. Behav. 20, 209-221.

Froese, T. (2016). Interactivity should aim to extend, not reject, the conceptual foundations of enaction. Constructivist Foundations, 11:2, 247-249.

Froese, T., and Di Paolo, E. A. (2011). The enactive approach: theoretical sketches from cell to society. Pragmatics Cogn. 19, 1-36.

Fortineau-Brémond, C. (2017). Corrélation et énaction : retour sur un phénomène linguistique incarné, processuel et distribué. Langage et énaction: problématiques, approches linguistiques et interdisciplinaires, Signifiances (Signifying), 1, 3, 5-24.

http://revues.clermont-universite.fr/index.php/Signifiances/issue/view/20

Fuchs, T. et De Jaegher, H. (2009). Enactive intersubjectivity: Participatory sense-making and mutual incorporation. Phenom Cogn Sci (2009) 8: 465-486.

Gallagher, S. (2005). How the Body Shapes the Mind. Oxford: Oxford University Press.

Gardiner, A. H. ([1932] 1951). The Theory of Speech and Language. Oxford, Clarendon Press.

Glasersfeld, E. von (1991). Distinguishing the observer: An attempt at interpreting Maturana. Methodologia 8, 57-68.

Goldberg, A. E. (1995). Constructions : A construction grammar approach to argument structure. Chicago, University of Chicago Press.

Goldberg, A. E. (2006). Constructions at Work. The Nature of Generalization in Language. Oxford, Oxford University Press.

Goody, J. (1979). La Raison graphique: La domestication de la pensée sauvage. Paris, Editions de Minuit.

Grégoire, M. (2012). Le lexique par le signifiant. Méthode en application à l'espagnol. Sarrebruck, Presses Académiques Francophones.

Grégoire, M. (2014). Théorie de la Saillance submorphologique et neurosciences cognitives, In A. Eliman (dir.). Enonciation et neurosciences cognitives, Synergies Europe, $n^{\circ} 9$, Gerflint, 107-119.

Grégoire M. (2017). Towards an enactive lexicology : From muscle salience to signifying. Langage et énaction: problématiques, approches linguistiques et interdisciplinaires, Signifiances (Signifying), 1, 3, 67-88.

http://revues.clermont-universite.fr/index.php/Signifiances/issue/view/20

Guentchéva, Z. (1994). Manifestations de la catégorie du médiatif dans les temps du français. Langue française, 102, 8-23.

Guillaume, G. (1964). Langage et science du langage. Paris, Québec, Nizet / P. U. Laval.

Jakobson, R. (1963). Essais de linguistique générale. Tome I : les fondations du langage. Paris, Editions de Minuit.

Goffman, E. (1987). Façons de parler. Traduit de l'anglais par Alain Kihm. Paris, Minuit.

Guillaume, G. (2003). Prolégomènes à la linguistique structurale 1, Québec, PUL.

Guillaume, G. (2004). Prolégomènes à la linguistique structurale 2, Québec, PUL.

Guillaume, G. (2007). Essai de mécanique intuitionnelle 1, Espace et temps en pensée commune et dans les structures de langue. Québec, PUL. 
Guiraud, P., (1986 [1967]). Structures étymologiques du lexique français. Paris, Payot, rééd. Paris, Larousse.

Harvey, M. I. (2015). Content in languaging: why radical enactivism is incompatible with representational theories of language. Language Sciences, 48, 90-129.

Harvey, M. I., Gahrn-Andersen, R. et Steffensen, S. V. (2016). Interactivity and Enaction in Human Cognition. Constructivist Foundations 11 (2), 234-245.

Hofstadter, D. et Sander, E. (2013). L’analogie, le coeur de la pensée. Paris, O. Jacob.

Honeste, M.-L. (2011). Le sens des mots entre langue et discours. In A. Ouattara (éd.), La linguistique de Bernard Pottier: bilan, critiques, perspectives. Rennes, Presses Universitaires de Rennes, coll. Rivages Linguistiques, 324-345.

Honeste, M.-L. (2015). Une approche expérientielle de la sémantique lexicale. Intellectica, 2015/2, 64, 87-112.

Humboldt, W. von (1836/1974). La différence de construction du langage dans l'humanité et l'influence qu'elle exerce sur le développement spirituel de l'espèce humaine ou Introduction à l'œuvre sur le kav. In: Introduction à l'œuvre sur le kavi et autres essais (Traduction de l'allemand par P. Caussat). Paris, Seuil, 143-419.

Hutchins, E. (2010). Enaction, imagination, and insight, in J. Stewart, O. Gapenne, and E. A. Di Paolo (eds). Enaction: Toward a New Paradigm for Cognitive Science. Cambridge, MA, The MIT Press, 425-450.

Itkonen, E. 1997. The Social Ontology of Linguistic Meaning. SKY: The Yearbook of the Linguistic Association of Finland, 49-80.

Itkonen, E. 2005. Analogy as Structure and Process: Approaches in Linguistics, Cognitive Psychology and Philosophy of Science. Amsterdam, Benjamins.

Jousse, M. (2008). L'anthropologie du geste. Paris, Gallimard, TEL.

Kenny, V. (1985). Life, the Multiverse and Everything : an Introduction to the Ideas of Humberto Maturana. http://www.oikos.org/vinclife.htm.

Kenny, V. (2007). Distinguishing Ernst von Glasersfeld's Radical Constructivism from Humberto Maturana's Radical Realism. Constructivist Foundations 2, 2-3, 58-64.

Kenny, V. (2009). There's Nothing Like the Real Thing. Revisiting the Need for a Third-Order Cybernetics. Constructivist Foundations 4, 2, 100-111.

Kerbrat-Orecchioni, C. (2005). Le discours en interaction. Paris, A. Colin.

Kravchenko, A. V. (2003). The ontology of signs as linguistic and non-linguistic entities: a cognitive perspective. Annual Review of Cognitive Linguistics 1, 179-191.

Kravchenko, A. V. (2007). Essential properties of language, or, why language is not a code. Language Sciences, 29, 650-671.

Kravchenko, Alexander V. (2011a). The semantics vs. pragmatics debate in the context of the orientational function of language. In A. Kiklewicz (dir.). Język poza granicami języka II. Semantyka a pragmatyka: spór o pierwszeństwo . Uniwersytet Warminsko-Mazurski w Olsztyne, 11-23.

Kravchenko, A. V. (2011b). How Humberto Maturana's biology of cognition can revive the language sciences. Constructivist Foundations 6, 3, 352-362.

Kravchenko, A. V. (2012a). Languaging as a consensual domain of coordinated interactions. Kaliningrad, $5^{\text {th }}$ International Conference on Cognitive Science.

Kravchenko, A. V. (2012b,). Grammar as semiosis and cognitive dynamics. In A. Kravchenko (éd.). Cognitive Dynamics in Linguistic Interactions. Cambridge Scholars Publishing, 125-153. 
Kravchenko, Alexander V. (2016). Two views on language ecology and ecolinguistics. Language Sciences, 54, 102-113.

Lafont, R. (1978). Le travail et la langue. Paris, Flammarion.

Lambrecht, K. (1994). Information Structure and Sentence Form. Topic, Focus and the Mental Representations of Discourse Referents. Cambridge University Press.

Launay, M. (1986). Effet de sens : produit de quoi ? Langages, 82, 13-39.

Lavie, R-J. (2003). Le locuteur analogique ou la grammaire mise à sa place, thèse de l'Université Paris Ouest Nanterre.

Lavissière, M. C., Loevenbruck, H. (2016). P300 and lexical decision about phonaesthemes. communication au colloque international Langage et énaction: Production $d u$ sens, Incarnation, Interaction, Université Clermont Auvergne, Clermont-Ferrand, 1-3 juin 2016.

Lacheret, A. et Beaugendre, F. (1998). La prosodie du français. Paris, CNRS Editions.

Lallot, J. (1988). Origines et développement de la théorie des parties du discours en Grèce. Langages 92, Les parties du discours, 11-23.

Lavelle, L. (1942-2007). La Parole et l'écriture. L'Artisan du livre (1942) ; Paris, Le Félin (2007).

Lenay, C. (2006). Énaction, Externalisme et Suppléance Perceptive. Intellectica, 2006/1, 43, 27-52.

Leroi-Gourhan, A. (1964). Le geste et la parole. I. Technique et langage. II. La mémoire et les rythmes. Paris, Albin Michel.

Le Tallec-Lloret, G. (2017). Linguistique du signifiant: diachronie et synchronie de l'espagnol. Limoges, Lambert-Lucas.

Levelt, W. (1989). Speaking : from intention to articulation, The MIT Press.

Liberman, A. M., Mattingly, I. G. (1985). The motor theory of speech perception revised. Cognition, $21,1-36$.

Linell, P. (2005). The Written Language Bias in Linguistics. Londres, Routledge.

Linell, P. (2009). Rethinking Language, Mind, and World Dialogically. Charlotte, Information Age.

Linell, P. (2012). On the Nature of Language : Formal Written-Language-Biased Linguistics vs. Dialogical Language Sciences. In A. Kravchenko (ed.). Cognitive Dynamics in Linguistic Interactions. Newcastle upon Tyne, Cambridge Scholars Publishing, 107-124.

Linell, P. (2013a). Distributed language theory, with or without dialogue. Language Sciences 40, $168-173$

Linell, P. (2013b). Are Natural Languages Codes? In Proceedings from Symposium on Language Acquisition and Language Evolution, PERILUS 2011 (Working Papers from the Phonetics Laboratory at the Department of Linguistics). Stockholm University, 39-50.

Love, N. (2004). Cognition and the language myth. Language Sciences 26 (6), 525-544

Love, N., (2007). Are languages digital codes? Language Sciences, 29 (5), 690-709.

Love, N. (2017). On languaging and languages. Language Sciences, 61, May 2017, 113-147.

Luhmann, N. (1990). Essays on Self-Reference. Columbia University Press.

Luhmann, N. (1995). Social Systems. Stanford, CA, Stanford University Press.

Macchi, Y. (2000). L'anticipation syntaxique de l'attribut. Essai de chronosyntaxe. In A. Résano (éd.) Linguistique hispanique. Actes du VIIIème Colloque de Linguistique Hispanique. Nantes : CRINI, 395-413.

Macchi, Y. (2006). Transitivité et intransitivité : propriétés du mot ou effets du processus phrastique ? Chronosyntaxe (VI). In G. Luquet (éd.). Le signifié de langue en espagnol. Paris, Presses Sorbonne Nouvelle, 115-134.

Macchi, Y. (2008). On vous le ramènera, votre mari!: Esquisse d'une topologie du signifié. Chronosyntaxe (III). Chréode, 1, Paris IV, Éditions Hispaniques, 141-178.

Mattia-Viviès, M. de (éd). (2012). La syntaxe mensongère. E-rea, 9-2, http://journals.openedition.org/erea/2323

Maturana, H. (1978). Biology of language: The epistemology of reality. In G. Miller, and E. Lenneberg (eds.). Psychology and Biology of Language and Thought: Essays in Honor of Eric Lenneberg. New York: Academic Press, 27-64. 
Maturana, H. (1987). Everything is said by an observer. In W. Thompson (éd.). Gaia, a Way of Knowing. Great Barrington, Lindisfarne Press, 65-82.

Maturana, Humberto. (1995). The nature of time. Santiago de Chile : Instituto de Terapia Cognitiva. [en ligne]

Maturana, H. R. et Varela, F.J. (1980). Autopoiesis and cognition: the realization of the living. Reidel, Dordrecht.

Maturana, H. et Varela, F.J. (1987). The tree of knowledge. Boston, Shambhala.

McGurk, H., McDonald, J. (1976). Hearing lips and seeing voices. Nature, 264, 23-30.

Mead, G. H. (1934/2006). L'esprit, le soi et la société (Traduction de l'américain par D. Cefaï et L. Quéré). Paris, PUF.

Medvedev, P. (1928/2008). La méthode formelle en littérature. Introduction à une poétique sociologique (Edition critique et traduction du russe par B. Vauthier et R. Comtet). Toulouse, Presses Universitaires du Mirail.

Menary, R. (2007). Writing as thinking. Language Sciences 5, 621-632.

Merleau-Ponty, M. (1945). Phénoménologie de la perception. Paris, Gallimard.

Molho, M. (1988). L'Hypothèse du formant (sur laconstitution du signifiant : esp. UN/UNO). In C. Blanche-Benveniste, A. Chevel \& M. Gross (éds.), Hommage à la mémoire de Jean Stefanini, Aix-en-Provence, Université de Provence, 291-303.

Mondada, L. (2005). Chercheurs en interaction. Comment émergent les savoirs. Lausanne, Presses Polytechniques et Universitaires Romandes.

Monneret, P. (2003). Le sens du signifiant. Implications linguistiques et cognitives de la motivation. Paris, Champion.

Monneret, P. (2004). Essais de linguistique analogique. Dijon, ABELL.

Nemo, F., (2006). Éléments pour une typologie linguistique des rapports forme/sens. Cahiers de linguistique analogique, 2, 205-227.

Neyrod, D. (2017). « Discours sur le mot » et « discours du mot » : la dialectique perplexe du signe et de l'objet. L'exemple du mot castillan sacre. Langage et énaction: problématiques, approches linguistiques et interdisciplinaires, Signifiances (Signifying), 1, 3, 171-181. http://revues.clermont-universite.fr/index.php/Signifiances/issue/view/20

Nobile, L. (2008). The Grammatical Monophonemes of Standard Italian. A Structural Isomorphism between Phonological and Semantic Oppositions ? Cognitive Philology, I-2.

Noë, A (2004). Action in Perception. Cambridge, MA, MIT Press.

Noë, A. (2009). Out of our Heads: Why You are not Your Brain, and other Lessons from the Biology of Consciousness. London, Macmillan.

Núñez, R., et Sweetser, E. (2006). With the Future Behind Them: Convergent Evidence From Aymara Language and Gesture in the Crosslinguistic Comparison of Spatial Construals of Time. Cognitive Science, 30(3), 401-450.

Ogien, A. (2007). Les formes sociales de la pensée, La sociologie après Wittgenstein. Paris, Colin.

Olivier, G. (2011). La cognition gestuelle. Grenoble, PUG.

Olivier G. et Bottineau D. (2015). Gestural Dimension of the Perceptuomotor Compatibility Effect in the Speech Domain. Swiss Journal of Psychology 74(2), Apr. 2015, 105-110.

O'Regan, J. K., et Noë, A. (2001). A sensorimotor account of vision and visual consciousness. Behavioral and Brain sciences, 24(05), 939-973.

Oudeyer, P.-Y. (2006). Self-organization in the evolution of speech. Oxford.

Pagès, S. (2017). Analysis of the SER/ESTAR opposition based on the origins and evolution of \{st\} salience: a submorphological and enactive approach. Langage et énaction: problématiques, approches linguistiques et interdisciplinaires, Signifiances (Signifying), 1, 3, 89-100.

http://revues.clermont-universite.fr/index.php/Signifiances/issue/view/20

Peirce, C. S. [1894]. What is a Sign? In Houser and Kloesel (eds). The Essential Peirce. Selected Philosophical Writings. Vol 2. (1893-1913). Indiana University Press, 4-10. 
Penelaud, O. (2010). Le paradigme de l'énaction aujourd'hui, Apports et limites d'une théorie cognitive révolutionnaire». PLASTIR, 2010/1, 18,

http://plasticites-sciencesarts.org/Plastir18_fr.html

Petitmengin, C. (2017). Enaction as a Lived Experience: Towards a Radical Neurophenomenology. Constructivist Foundations 12(2), 139-147.

Philps, D. (2002). Le concept de 'marqueur sub-lexical' et la notion d'invariant sémantique. In P. Larrivée (dir). La notion d'invariant sémantique, Travaux de linguistique, 45, 103-123.

Philps, D. (2006). From mouth to hand. Proceedings of the 6th International Conference on the Evolution of Language, 247-254.

Philps, D. (2008). Submorphemic iconicity in the lexicon: a diachronic approach to English gnwords. Lexis, E-Journal in English Lexicology.

Philps D. (2017). The emergence of the linguistic sign : vocomimesis, symmetry and enaction. Langage et énaction: problématiques, approches linguistiques et interdisciplinaires, Signifiances (Signifying), 1, 3, 115-132.

http://revues.clermont-universite.fr/index.php/Signifiances/issue/view/20

Piotrowski, D. (2012). Sur la 'concrétude' du signe: Saussure et Husserl, Linguistique et phénoménologie du langage. La Tribune Internationale des Langues Vivantes, 52-53, 30-43.

Plantin, C., Doury, M., Traverso, V. (2000). Les émotions dans les interactions. Lyon, Presses Universitaires de Lyon.

Poirier, M. (à paraître a). La grammaticalisation par le signifiant: le cas de cualquier. Submorphémie, réseaux et émergence du sens. In C. Fortineau-Brémond et É. Blestel (éds.). Le signifiant sens dessus dessous. Submorphémie et chrono-analyse en linguistique hispanique. Limoges, Lambert-Lucas.

Poirier, M. (à paraître b). También / tampoco : émergence d'un micro-système par le signifiant. Submorphémie, diachronie et chronosignifiance. In S. Pagès (éd.) Submorphologie et diachronie dans les langues romanes. Aix-en-Provence, Presses Universitaires de Provence.

Poirier, M. (2017). Esquisse des principes d'une chronosignifiance. Langage et énaction: problématiques, approches linguistiques et interdisciplinaires, Signifiances (Signifying), 1, 3, 41-66. http://revues.clermont-universite.fr/index.php/Signifiances/issue/view/20 .

Pöppel, E. (1998). Wie kommt die Zeit in den Kopf? Zeitschrift für Kultur Austausch, 3, 29-31.

Priscien (2010). Grammaire. Livre XVII - Syntaxe I/ Paris, Vrin.

Raimondi, V. (2014a). Social interaction, languaging and the operational conditions for the emergence of observing. Frontiers in Psychology, 5:899.

Raimondi, V. (2014b). Interaction, coordination, languaging: la matrice opérationnelle-relationnelle du langage. Intellectica, 2014/2, 62, 35-49.

Rastier, F. (1987). Sémantique interprétative. Paris, PUF.

Rastier, F. (1996). Représentation ou interprétation ? - Une perspective herméneutique sur la médiation sémiotique. In V. Rialle et D. Fisette (éds), Penser l'esprit : des sciences de la cognition à une philosophie de l'esprit. Grenoble, Grenoble, Presses Universitaires de Grenoble, 219-239.

Rastier, F. (2005). Herméneutique et linguistique : dépasser la méconnaissance. Texto! [en ligne], décembre 2005, vol. X, n4. $<$ http://www.revue-texto.net/Dialogues/Debat_Hermeneutique/Rastier_Herm-et-ling.html>.

Rastier, F. (2006). De la signification lexicale au sens textuel : éléments pour une approche unifiée. Texto! [en ligne], mars 2006, vol. XI, $\mathrm{n}^{\circ} 1$. Disponible sur : $<$ http://www.revue-texto.net/Inedits/Rastier/Rastier_Signification-lexicale.html>. (Consultée le 23/08/2017)

Rastier, F. (2011). Langage et pensée : dualisme cognitif ou dualité sémiotique ? Intellectica, 2011/2, 56, 29-79.

Reinmuth, J.-C. (2017). La mère, le sein, la tétée, l'amour et le baiser : une analyse diachronique en soutien des théories de l'énaction et de l'incarnation. Langage et énaction : problématiques, 
approches linguistiques et interdisciplinaires, Signifiances (Signifying), 1, 3, 133-149.

http://revues.clermont-universite.fr/index.php/Signifiances/issue/view/20

Robert, S. (éd.). (2003). Perspectives synchroniques sur la grammaticalisation. Peeters, Louvain-Paris.

Rosier, L. (1995). L’interjection, partie 'honteuse' du discours. Scolia 3, 109-121.

Rosenfield, I. (1994). L'invention de la mémoire. Paris, Flammarion.

Ross, D. (2007). H. sapiens as ecologically special: what does language contribute? Language Sciences, 29/5: 710-731.

Roy, J.-M. (2013), Pragmatisme cognitif et énactivisme. Intellectica, 2013/2, 60, 69-89.

Rovelli, C. (2014). Et si le temps n'existait pas? Paris, Dunod,

Simondon, G. (1989). L'individuation psychique et collective. Paris, Aubier.

Smith, C. (2015). The phonesthetics of blends ; a lexicographic study of cognitive blends in the OED. Exploration in English Language and Linguistics, ExELL 2/1. 4/5/2015. http://www.exell.ff.untz.ba/?issue=volume-2-1

Smith, C. (2016). Tracking semantic change in fl- monomorphemes in the OED. Journal of Historical Linguistics 6:2 (2016). Amsterdam: John Benjamins, 165-200.

Sperber, D., Wilson, D. (1995). Relevance. Communication and Cognition (2nd edition). Oxford (UK) et Cambridge (USA), Blackwell.

Saussure, F. de (2002). Ecrits de linguistique générale, S. Bouquet et R. Engler (eds). Paris, Gallimard.

Simon, A.-C. et Christodoulides, C. (2016). Frontières prosodiques perçues : corrélats acoustiques et indices syntaxiques. In M. Avanzi, A.-C. Simon, B. Post (éds). La prosodie du français : accentuation et phrasé, Langue française 191, 2016/3, 83-106.

Spinoza, B. (1677/2005). Ethique (Traduction du latin et commentaires par R. Misrahi). Paris, Editions de l'Eclat.

Steffensen, S. V., et Fill, A. (2014). Ecolinguistics: the state of the art and future horizons. Language Sciences, 41, 6-25.

Stewart, J. (1996). Cognition = Life: Implications for higher-level cognition. Behavioural Processes 35, 311-326.

Stewart, J. (2001). Le sens biologique. In D. Keller, J.-P. Durafour, J. F. P. Bonnot et R. Sock (éd.). Percevoir : monde et langage. Invariance et variabilité du sens vécu. Mardaga, Sprimont, 117-127.

Stiegler, B. (1994). La Technique et le temps 1 : La faute d'Epiméthée. Paris, Galilée.

Swenson, R. (1992). Autocatakinetics, Yes - Autopoiesis, No: Steps Toward a Unified Theory of Evolutionary Ordering. Int. J. General Systems. 21, 207-208.

Swiatkowska, M. (2000). Entre dire et faire. De l'interjection. Cracovie, Wydawnictwo Uniwersytetu Jagiellonskiego.

Tesnière, L. (1969). Eléments de Syntaxe structurale. Paris, Klincksieck.

Thibault, P. J. (2011). First-Order Languaging Dynamics and Second-Order Language: The Distributed Language View. Ecological Psychology, 23, 1-36.

Tollis, F. (2008). La linguistique de Gustave Guillaume : de l'opérativité à la socio-opérativité ? Cahiers de praxématique [En ligne], 51 | 2008, mis en ligne le 01 janvier 2013, consulté le 18 décembre 2015. URL : http://praxematique.revues.org/1073

Tollis, F. (2017). La théorie linguistique de Gustave Guillaume scrutée dans une perspective énactive. Langage et énaction : problématiques, approches linguistiques et interdisciplinaires, Signifiances (Signifying), 1, 1, 39-49.

http://revues.clermont-universite.fr/index.php/Signifiances/issue/view/20

Tomatis, A. (1991). Nous sommes tous nés polyglottes. Paris, Fixot.

Tournier, J. [1985] (2007). Introduction descriptive à la lexicogénétique de l'anglais contemporain. Paris-Genève, Champion-Slatkine.

Toussaint, M. (1983). Contre l'arbitraire du signe. Paris, Didier Érudition. 
Toussaint, M. (2003). Analogiques. In Ph. Monneret (éd.). Le mot comme signe et comme image : lieux et enjeux de l'iconicité linguistique, Cahiers de linguistique analogique, 1, Dijon, ABELL, 331-350.

Ubersfeld, A. (1977), Lire le théâtre. Paris, Éditions Sociales.

Valette, M. (2006). Linguistiques énonciatives et cognitives françaises : Gustave Guillaume, Bernard Pottier, Maurice Toussaint, Antoine Culioli. Paris, H. Champion.

Vion, R. (2014). Polyphonie énonciative et dialogisme, Actes du colloque Dialogisme : langue, discours 2010. Praxiling UMR 5267 CNRS - Université Paul-Valéry - Montpellier 3, http://www.praxiling.fr/dialogisme-langue-discours.html

Vion-Dury, J., Besson, M., Cermolacce, M., Schön, D. \& Piotrowski, D. (2015). Neurophénoménologie du signe linguistique : Apport du modèle Phénoménologique Morphodynamique et Structuraliste (PMS) à la compréhension des mécanismes neuraux sous-tendant la donation de sens. Intellectica, 2015/2, 64, 123-157.

Volochinov, V. N. (1929/2010). Marxisme et philosophie du langage. Les problèmes fondamentaux de la méthode sociologique dans la science du langage (Traduction du russe par P. Sériot et I. Ageeva-Tylkowski). Limoges, Lambert-Lucas.

Von Uexküll, J. (2010.) A foray into the world of animals and humans; with a theory of meaning. Minneapolis: University of Minnesota Press.

Vygotski, L. S. (1934/1997). Pensée et langage (Traduction du russe par F. Sève; $3^{\mathrm{e}}$ éd.). Paris, La Dispute. 